\title{
Wilms tumor 1 (WT1) regulates KRAS-driven oncogenesis and senescence in mouse and human models
}

\author{
Silvestre Vicent, ${ }^{1}$ Ron Chen, ${ }^{1}$ Leanne C. Sayles, ${ }^{1}$ Chenwei Lin, ${ }^{1}$ Randal G. Walker,,${ }^{1,2}$ \\ Anna K. Gillespie, ${ }^{1}$ Aravind Subramanian, ${ }^{3}$ Gregory Hinkle, ${ }^{3}$ Xiaoping Yang, ${ }^{3}$ Sakina Saif, ${ }^{3}$ \\ David E. Root, ${ }^{3}$ Vicki Huff, ${ }^{4}$ William C. Hahn, ${ }^{3,5}$ and E. Alejandro Sweet-Cordero'
}

${ }^{1}$ Cancer Biology Program, Department of Pediatrics, Stanford University School of Medicine, Stanford, California, USA. ${ }^{2}$ CardioDx, Palo Alto, California, USA ${ }^{3}$ Broad Institute of Harvard and MIT, Cambridge, Massachusetts, USA. ${ }^{4}$ Department of Genetics, MD Anderson Cancer Center, Houston, Texas, USA.

${ }^{5}$ Dana Farber Cancer Institute, Department of Medical Oncology, Boston, Massachusetts, USA.

\begin{abstract}
KRAS is one of the most frequently mutated human oncogenes. In some settings, oncogenic KRAS can trigger cellular senescence, whereas in others it produces hyperproliferation. Elucidating the mechanisms regulating these 2 drastically distinct outcomes would help identify novel therapeutic approaches in RAS-driven cancers. Using a combination of functional genomics and mouse genetics, we identified a role for the transcription factor Wilms tumor 1 (WT1) as a critical regulator of senescence and proliferation downstream of oncogenic KRAS signaling. Deletion or suppression of Wt1 led to senescence of mouse primary cells expressing physiological levels of oncogenic Kras but had no effect on wild-type cells, and Wt 1 loss decreased tumor burden in a mouse model of Kras-driven lung cancer. In human lung cancer cell lines dependent on oncogenic KRAS, WT1 loss decreased proliferation and induced senescence. Furthermore, WT1 inactivation defined a gene expression signature that was prognostic of survival only in lung cancer patients exhibiting evidence of oncogenic $K R A S$ activation. These findings reveal an unexpected role for WT1 as a key regulator of the genetic network of oncogenic KRAS and provide important insight into the mechanisms that regulate proliferation or senescence in response to oncogenic signals.
\end{abstract}

\section{Introduction}

Mutations in KRAS are commonly found in pancreatic, lung, and colon cancers (1). Furthermore, many cancers expressing wildtype KRAS have genetic alterations in genes known to function in the RAS pathway $(2,3)$. Thus, understanding the mechanisms responsible for $K R A S$-induced oncogenesis is an important goal in cancer research. While several RAS-effector pathways are well known, aberrant RAS signaling leads to alterations in a vast and only partially understood signaling network involving both forward activation via kinase cascades and negative feedback mediated by transcriptional regulation $(4,5)$. The importance of dissecting the complexity of this network is demonstrated by the dramatically distinct consequences of oncogenic RAS mutations depending on the cellular context. Activation of the RAS pathway can lead to either proliferation or senescence $(6,7)$. However, the precise mechanisms governing these distinct outcomes are not fully understood.

An important consequence of signaling downstream of RAS is a change in the expression of a large number of genes. Previous work has identified KRAS-specific gene expression signatures using mouse, zebrafish, or human model systems (8-11). Gene expression signatures are useful tools for identifying the complex signaling networks that drive diverse cellular processes in normal physiology and disease. For example, expression correlations between genes in microarray data have also been utilized to identify transcription factors that act as "master regulators"

Authorship note: Ron Chen and Leanne C. Sayles contributed equally to this work. Conflict of interest: The authors have declared that no conflict of interest exists. Citation for this article: J Clin Invest. 2010;120(11):3940-3952. doi:10.1172/JCI44165. of oncogenic transformation $(12,13)$. Such an approach has not been systematically applied to the elucidation of oncogenic KRAS-driven signaling networks.

Negative-selection RNAi screens are a powerful approach for high-throughput functional analysis of genes in mammalian systems (14-17). Recently, RNAi screens have been used to identify STK33, PLK1, and TBK1 as synthetic lethal in cells expressing oncogenic RAS (18-20). As genome-wide screening approaches generally are limited by low signal and high noise and are thus nonsaturating, a more focused approach based on querying the functional significance of defined subsets of genes could yield novel insight into RAS biology. To test this hypothesis, an shRNA-negative selection screen was used to query an oncogenic KRAS gene expression signature and its putative transcriptional regulators. This approach identified what we believe to be a novel role for Wilms tumor 1 (WT1) as specifically required in cells expressing oncogenic KRAS but not in cells expressing wild-type KRAS. The synthetic effect of WT1 loss in cells expressing oncogenic KRAS was tested and confirmed in primary cells, in a genetically engineered mouse model, and in human cell lines. In both mouse and human cells, loss of WT1 activated a senescence program in cells expressing oncogenic KRAS but not in cells expressing wild-type KRAS. Increasing evidence points to a critical role for senescence as a barrier to oncogene-induced tumorigenesis (21). The studies described here highlight WT1 as a key modulator of senescence driven by oncogenic KRAS and establish what we believe is a previously unknown link between a critical oncogenic signaling pathway and a gene otherwise best characterized for its role in development and in tumor suppression. 
A
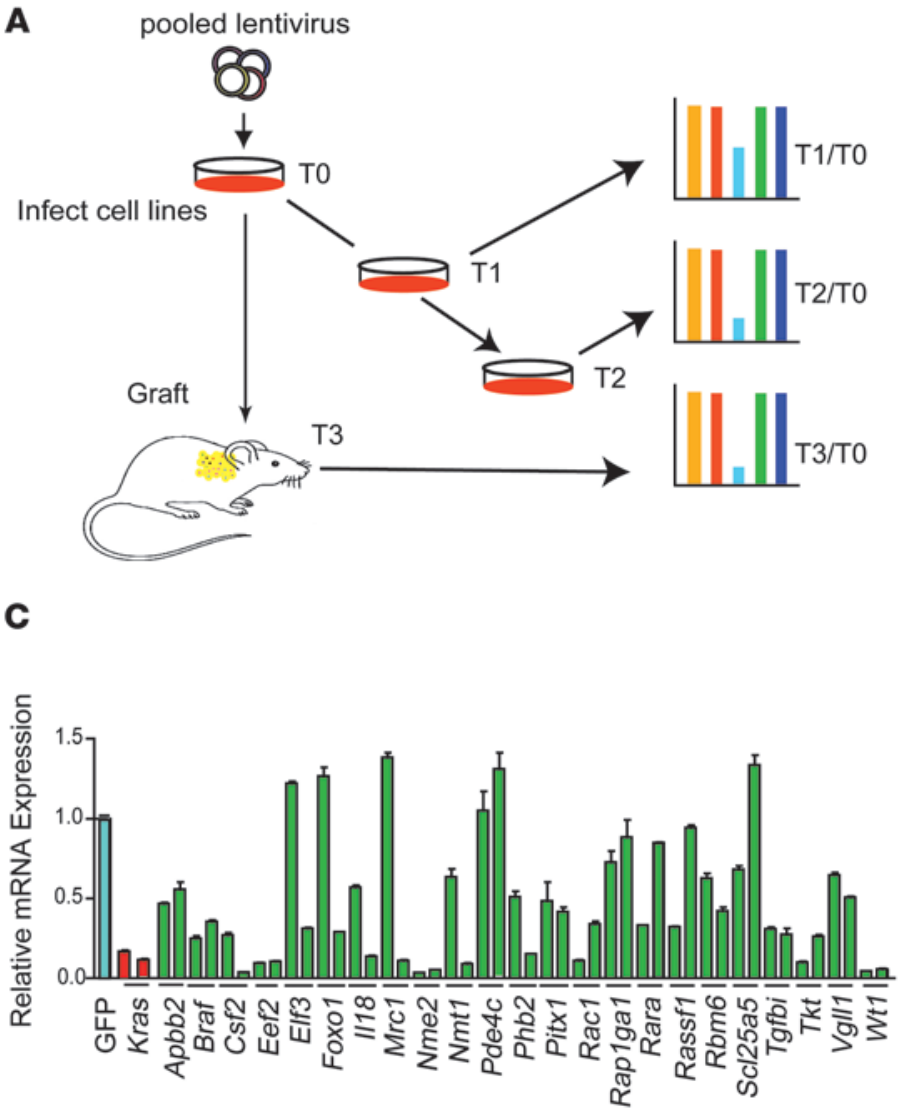

B

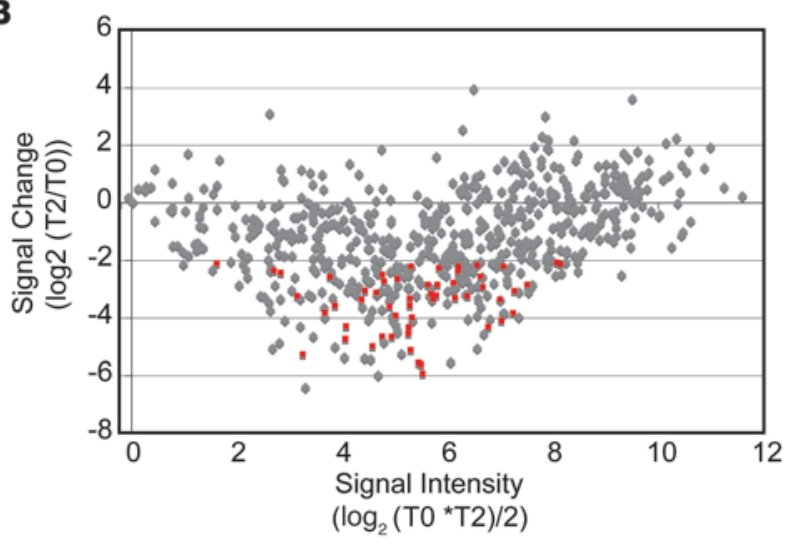

D

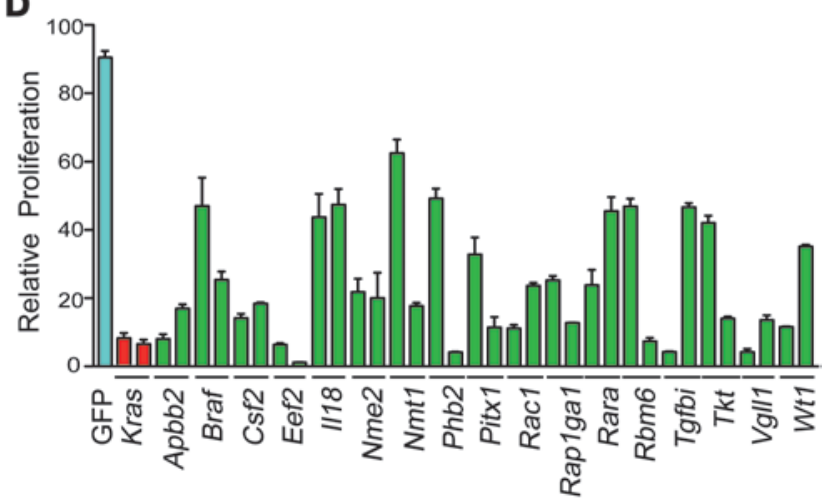

Figure 1

A negative selection screen to identify Kras effectors. (A) Design of shRNA screen. T1 and T2 show 3 and 6 weeks in vitro proliferation, respectively. T3 shows 3 weeks of subcutaneous growth. Colored bars represent the relative ratio of the MFI of a particular barcode corresponding to a shRNA at T1/T2/T3 over T0. Light blue bar depicts a theoretical barcode negatively selected over time. (B) Quantitation of each shRNA at T2 compared with T0. MFI measurements are average of 3 independent experiments done for each of the 2 cell lines at each time point. $x$ axis shows $\log _{2}$ of average mean MFI for each shRNA. $y$ axis shows $\log _{2}$ of fold change of MFI between the 2 time points. Red squares indicate shRNAs negatively selected as described in text, with at least 1 other negatively selected shRNA against the same gene. (C) Knockdown of intended target for each shRNA identified as negatively selected using rtPCR. Results show relative mRNA expression for each gene compared with a control shRNA. Results for knockdown of Kras are also shown. (D) Proliferation of LKR13 cells infected with shRNAs for target genes that showed on-target effect as demonstrated in C. Cells were place in 96-well plates and analyzed using the MTT assay at day 7 after selection. Results are the average of 3 independent measurements. Error bars indicate mean \pm SD.

\section{Results}

An RNAi screen identifies candidate participants in a genetic network required for oncogenic Kras function. A negative selection shRNA screen was carried out to identify genes required for Kras-driven oncogenesis. We included in the screen genes previously identified as part of a Kras gene expression signature $(11)(n=89)$ as well as potential transcriptional regulators identified using the KolmogorovSmirnov scanning (KSS) algorithm (13) $(n=35)$ (Supplemental Table 1; supplemental material available online with this article; doi:10.1172/JCI44165DS1). KSS uses microarray data to establish a correlation matrix between transcription factors ${ }_{1 \ldots n}$ and all other genes. This matrix is then used to identify enrichment of a given gene set within each list of transcription factor-correlated genes. In this analysis, a large compendium $(n=190)$ of gene expression microarray data from cancer tissues and cell lines was used to establish the correlation matrix (22). Transcription factors with high enrichment of Kras signature genes in their correlated gene lists were included in the screen (see Supplemental Methods). shRNAs against other genes were introduced in the library because they had been previously implicated as Kras effectors $(n=47)$ or because they were differentially expressed in prior microarray experiments performed on human non-small cell lung cancer (NSCLC) samples $(n=20)$ or cell lines $(n=23)$ (see Supplemental Methods and Supplemental Table 2). A primary goal was to identify shRNAs that confer a disadvantage to the proliferation of Kras mutant tumor cells and would thus be selected against in a pooled screen. To maximize the likelihood of identifying such shRNAs, a detection platform with high signal and low background was employed (23) (see Supplemental Methods). The performance of this system was first tested by analyzing the ability to detect negative selection of an shRNA against Kras (Supplemental Figure 1A).

Pooled vectors carrying shRNAs against target genes were used to produce virus (Supplemental Table 2) and infect mouse lung cancer cell lines LKR10 and LKR13 (24). Cells were allowed to proliferate in vitro or injected subcutaneously into immunodeficient mice (Figure 1A). The relative presence of each shRNA was quan- 


\section{Table 1}

List of 23 genes that scored as negatively selected in either the in vitro proliferation screen or the in vivo tumorigenesis screen

\begin{tabular}{lcc} 
Gene Symbol & Function & Screen scored \\
Apbb2 & Adaptor protein & Both \\
Braf & Protein kinase & Both \\
Csf2 & Cytokine & In vitro \\
Eef2 & Protein synthesis & Both \\
Elf3 & Transcription factor & Both \\
Foxo1 & Transcription factor & Both \\
IL18 & Cytokine & Both \\
Mrc1 & DNA replication fork machinery & Tumorigenesis \\
Nme2 & Histidine kinase & Both \\
Nmt1 & Myristoyl transferase & Both \\
Pde4c & cAMP nucleotide phosphodiesterase & Both \\
Pitx1 & Transcription factor & Both \\
Phb2 & Transcription repressor & Tumorigenesis \\
Rac1 & Small GTPase & Both \\
Rap1ga1 & GTPase-activating protein & Both \\
Rara & Transcription factor & Tumorigenesis \\
Rassf1 & Tumor suppressor & Both \\
Rbm6 & RNA-binding protein & Both \\
Slc25a5 & Transport molecule & In vitro \\
Tgfbi & Extracellular protein & In vitro \\
Tkt & Tyrosine kinase & Both \\
Vgll1 & Transcription factor coactivator & Both \\
Wt1 & Transcription factor & Both \\
& & \\
\hline Scen & &
\end{tabular}

Screen scored column indicates whether genes scored in the subcutaneous tumor growth screen (T3 vs. T0), in the in vitro proliferation screen (T2 vs. T0, and T1 vs. T0), or in both screens.

tified using fluorescent beads to which an shRNA-specific "barcode" had been attached (see Methods). Negatively selected shRNAs were defined as those with a statistically significant decrease in MFI at time point 1 (T1) and T2 (in vitro) or T3 (in vivo) when compared with T0 (Figure 1B, Supplemental Figure 1, B-D, and Supplemental Table 3). To control for off-target effects, only genes that met these criteria with 2 or more shRNAs were considered. The 23 genes identified as negatively selected are shown in Table 1. Importantly, the list includes 2 known Kras effectors (Braf and Rac1). Seventeen out of the 23 hits had 2 shRNAs that decreased the level of expression of the target by more than 50\% (Figure 1C). Those shRNAs without at least a 50\% decrease in expression of the intended target were considered likely to be off target and removed from further analysis. All on-target shRNAs demonstrated a profound impact on proliferation, confirming why they scored in the initial screen (Figure 1D).

A secondary screen identifies Wt1 as a critical component of oncogenic Kras signaling. To determine which of the previously identified genes might function as synthetic lethals in cells expressing oncogenic Kras, Kras ${ }^{L S L-G 12 D /+}$, or wild-type control, mouse embryo fibroblasts (MEFs) were infected with a pool of those shRNAs negatively selected in the initial screen (25). Relative changes over time for each shRNA were quantified as above (Figure 2A). Two shRNAs against 3 genes, Rac1, $P b b 2$, and $W t 1$, were negatively selected in Kras ${ }^{G 12 D /+}$ MEFs but not in wild-type MEFs (Figure 2B and Supplemental Figure 2). Rac1 has previously been linked to Ras signaling in lung cancer (26). Pbb2 is a mitochondrial chaperone protein that regulates responses to apoptotic stimuli (27). Wt1 is a gene with multiple isoforms and pleiotropic cellular functions that was originally identified as a tumor suppressor linked to the pathogenesis of some cases of Wilms tumor (28). While best characterized as a transcription factor, $W t 1$ has isoforms that do not bind DNA but have been implicated in RNA processing (29). Wt1 was included in the initial screen because it was identified as a potential transcriptional regulator of the Kras signature. Thus, the role of Wt 1 in Kras-driven oncogenesis was further explored.

To confirm that the oncogenic Kras-specific effect of Wt1 loss observed in MEFs was also seen in lung epithelial cells, the pooled secondary screen was repeated in an immortalized mouse lung epithelial cell line (MLE12) that expresses wild-type Kras (30). shRNAs against $W t 1$ were not negatively selected in MLE12 cells, whereas a parallel experiment in LKR10 and LKR13 demonstrated negative selection for the same 2 shRNAs (Figure 2C). The results suggest that the Wt1 shRNAs have a deleterious effect specific to cells that express oncogenic Kras.

To determine the effect of Wt 1 loss on tumorigenesis, 2 shRNAs against Wt1 were used to infect LKR13 cells and perform a xenograft experiment. Wt1 suppression significantly decreased the size of tumors when compared with controls and to a level similar to that caused by suppression of Kras (Figure 2D). Taken together, these results suggest that in both mouse primary fibroblasts and lung epithelial cells, Wt1 expression is required only in the context of oncogenic Kras but is dispensable in cells expressing wild-type Kras.

Wt1 loss leads to senescence in primary cells expressing oncogenic Kras. To further characterize the interaction between oncogenic Kras and $W t 1$, we employed mice carrying a conditional allele of Wt1 $\left(W t 1^{f / f}\right)(31)$. Expression of Cre recombinase in Wt1f/f cells leads to in-frame deletion of exons 8 and 9 , generating a shortened protein $\left(W t 1^{\Delta / \Delta}\right)$ lacking zinc fingers 2 and 3 that is severely compromised for DNA binding. Wt1 $1 / f$ mice were crossed to $\mathrm{Kras}^{L S L-G 12 D /+}$ mice to obtain K-ras ${ }^{L S L-G 12 D /+} ; \mathrm{Wt} 1^{\mathrm{f} / f} \mathrm{MEFs}$. Infection of these MEFs with adenoviral Cre (AdCre) led to expression of oncogenic Kras ${ }^{G 12 D}$ and loss of wild-type Wt1 (Supplemental Figure 3, A and B). Real-time RT-PCR (rtPCR) analysis revealed the expected decrease in $W t 1$ full-length mRNA levels in both $W t 1^{\Delta / \Delta}$ and $\mathrm{Kras}^{\mathrm{G} 12 \mathrm{D} /+}$; Wt1 ${ }^{\Delta / \Delta}$ MEFs compared with $\mathrm{Kras}^{\mathrm{G} 12 \mathrm{D} /+}$ MEFs (Figure 3, A and B). Cell-cycle analysis 7 days after infection demonstrated a marked decrease in the number of cells in $S$ phase in Kras $^{G 12 D /+} ; W t 1^{\Delta / \Delta}$ compared with $W t 1^{f / f}$ or $W t 1^{\Delta / \Delta}$ cells (Figure 3C). No differences in the number of apoptotic cells were detected (data not shown). A 3T3 assay confirmed the previous observation that $\mathrm{Kras}^{\mathrm{G} 12 \mathrm{D} /+} \mathrm{MEFs}$ are hyperproliferative (25). Loss of wildtype Wt1 expression had no effect on MEFs expressing wild-type Kras. In contrast, Kras ${ }^{G 12 D /+} ; W_{t 1}{ }^{\Delta / \Delta}$ MEFs demonstrated markedly attenuated cumulative population doubling (CPD) (Figure 3D). These results provide strong genetic evidence for an interaction between oncogenic Kras and Wt1 and rule out off-target effects that may have occurred in the shRNA functional screen.

Kras $^{G 12 D /+}$; Wt $1^{\Delta / \Delta}$ MEFs demonstrated a flattened morphology and stained positive for senescence-associated $\beta$-gal (SA- $\beta$ gal) (Figure $3 \mathrm{E}$ ). This was surprising, as it has been assumed that oncogenic Kras leads to senescence in MEFs only when the oncogene is expressed at high levels (25). A key protein involved in senescence of both human and mouse fibroblasts is promyelocytic leukemia $(\mathrm{PML})(32,33)$. A dramatic increase in the localization of $\mathrm{Pml}$ to nuclear bodies (PNBs) in $\mathrm{Kras}^{\mathrm{G} 12 \mathrm{D} /+}$; $\mathrm{Wt} 1^{\Delta / \Delta} \mathrm{MEFs}$ was detected (Figure $3 \mathrm{~F}$ ). At this time point, we also observed a significant decrease in the number of BrdU-positive cells and a decrease in the number 
A

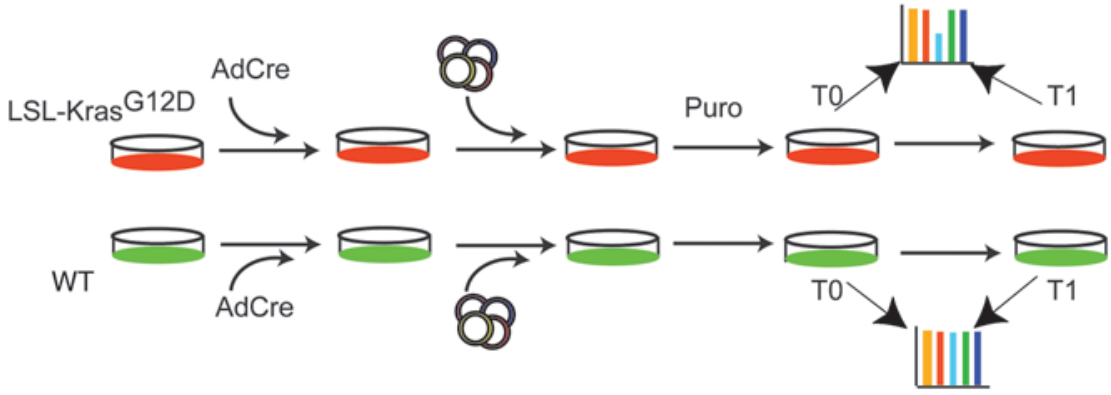

B

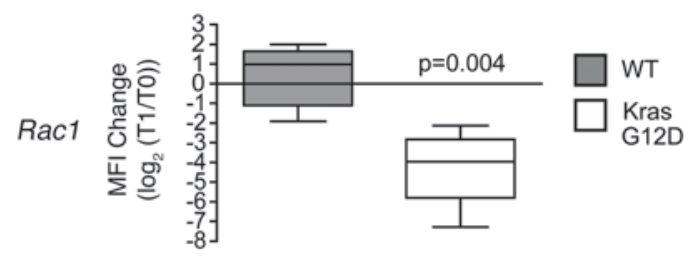

Phb2
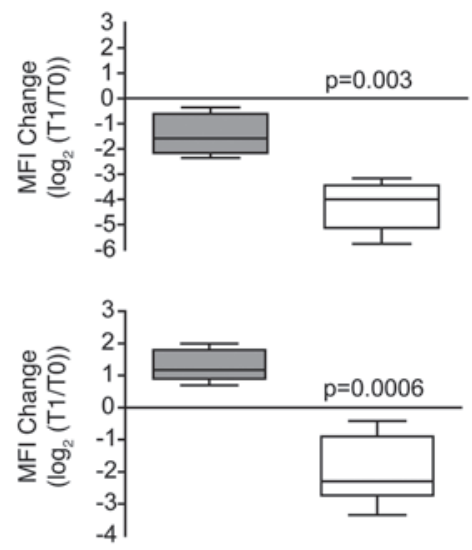

C

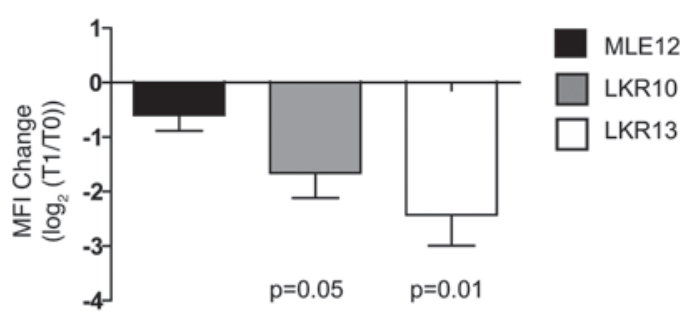

Wt1 shRNA 2

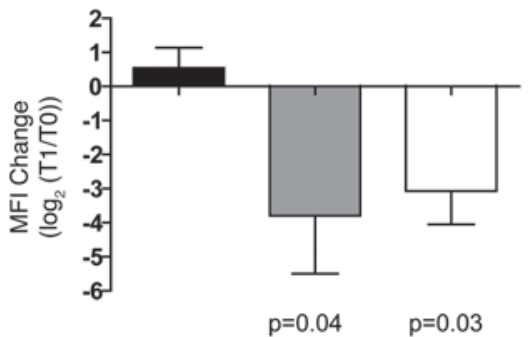

Wt1 shRNA 1

LE12

KR10

KR13

D

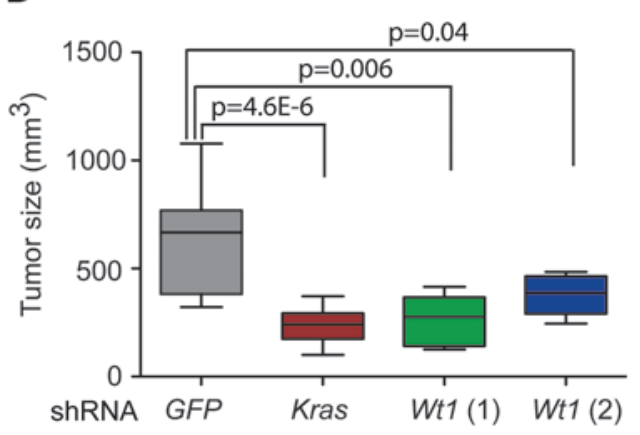

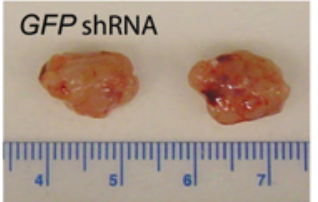

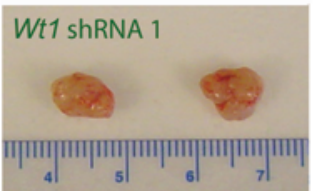

\section{Figure 2}

Identification of Wt1 loss as a Kras-specific synthetic-lethal interaction in MEFs. (A) Design of MEFs validation screen. MEFs were first infected with AdCre followed by infection with lentiviral vectors containing pools of shRNAs. 5 independent MEF lines of each genotype were assessed with each shRNA pool. The colored bars represent the relative ratio of the MFI of a particular barcode corresponding to a shRNA at T1, T2, or T3 over T0. The light blue bar depicts a theoretical barcode that is negatively selected. (B) Rac1, Phb2, and Wt1 are negatively selected in Kras $L S L-G 12 D /+M$ MEFs. Box plots indicate mean \pm SD of $\log _{2}$ of the MFI fold change for each gene comparing T1 versus T0. $P$ values were obtained using a 2-tailed $t$ test. (C) $W t 1$ shRNAs are not negatively selected in transformed lung epithelial cell lines expressing wild-type Kras. MLE12 cells were infected with the same shRNA pools used for the MEF experiments. $\log _{2}$ of the MFI fold change in the presence of 2 Wt1 shRNAs after 3 weeks of proliferation in vitro was measured and compared with the results for LKR10 and LKR13. Error bars show mean \pm SD. (D) LKR13 cells were infected with shRNAs to GFP (control), Kras, and Wt1, selected for 3 days, and injected subcutaneously $(n=8)$ into Balb/cnu/nu mice. Results show tumor volume at final day of experiment (day 24 after injection). Representative tumors are shown. Error bars indicate mean \pm SEM. All $P$ values are for a 2 -tailed $t$ test. 
A
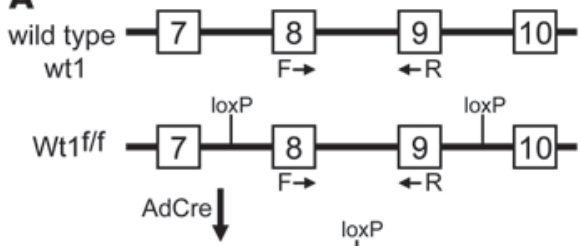

$\mathrm{Wt}^{\Delta / \Delta}$

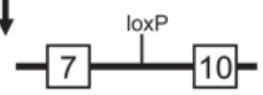

B

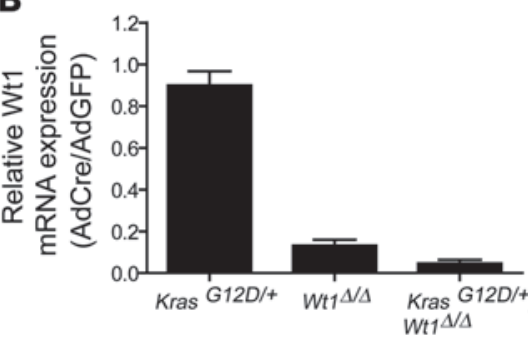

D
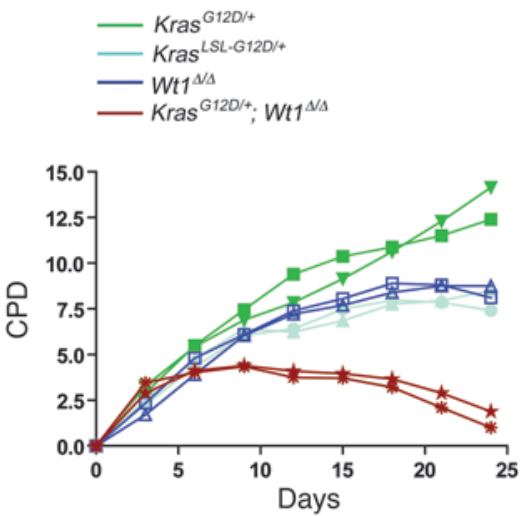
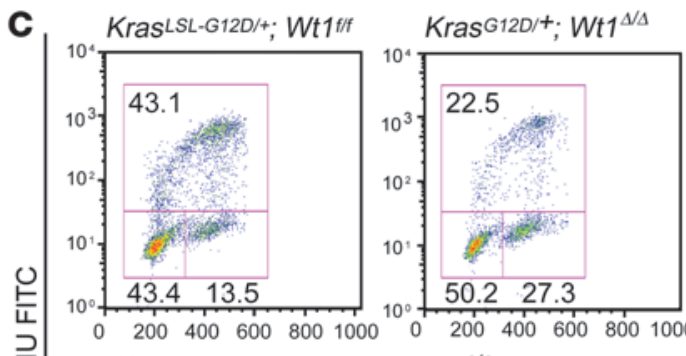

妾

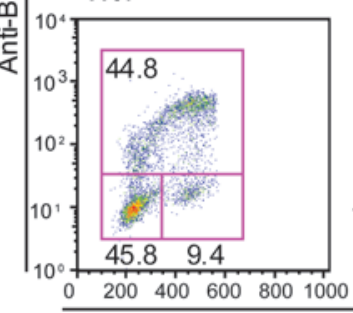
$W t 1^{\Delta / \Delta}$
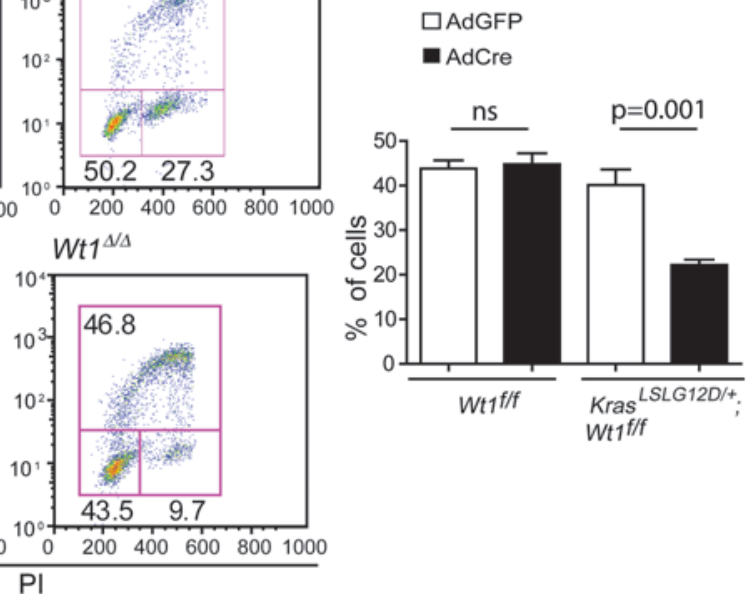

E

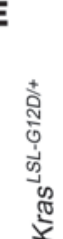

AdGFP

AdCre

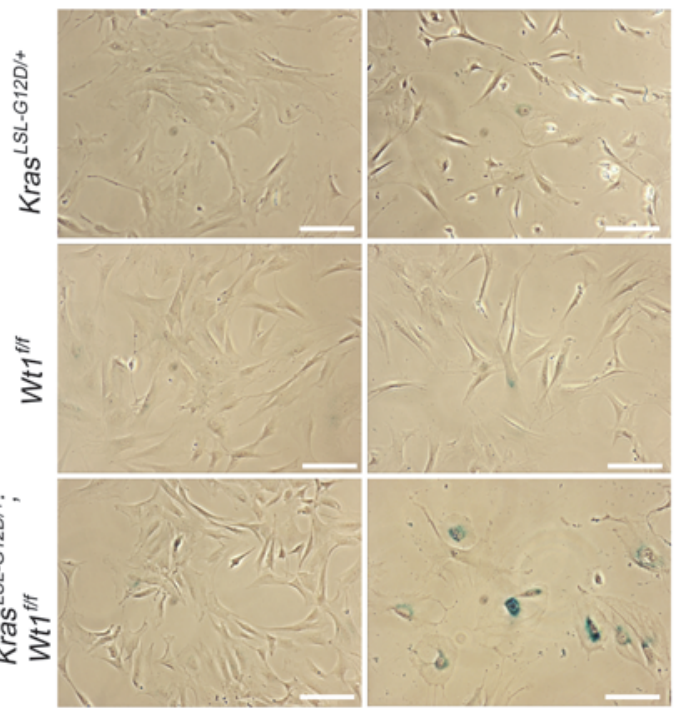

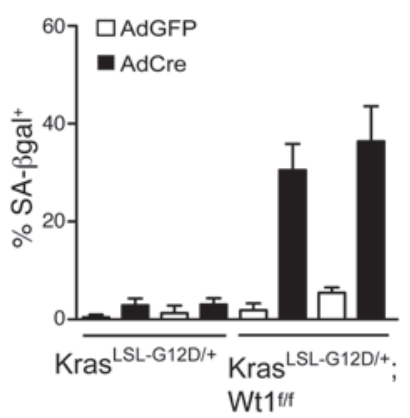

F
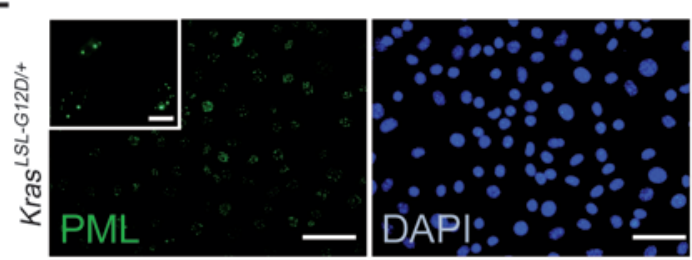

$\frac{5}{3}$
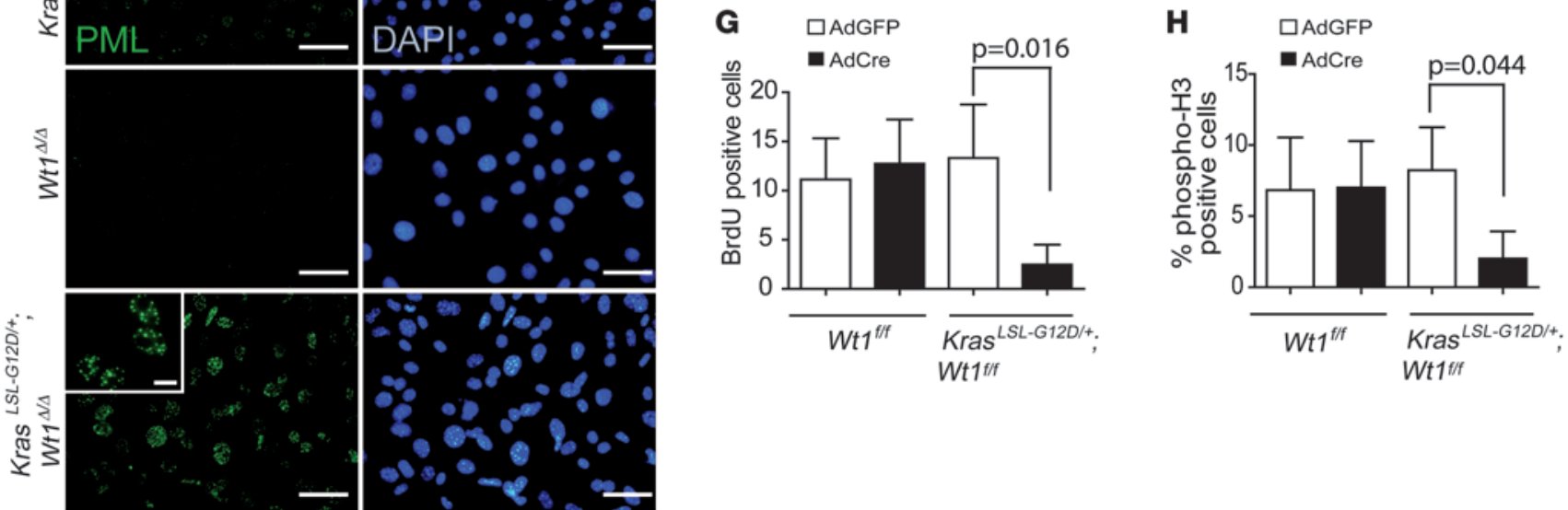


\section{Figure 3}

Deletion of Wt1 leads to senescence in primary cells expressing oncogenic Kras. (A) Primers for rtPCR were designed to amplify exons 8 $(\mathrm{F})$ and $9(\mathrm{R})$ of wild-type and conditional Wt1 alleles, and span intronic sequence. (B) rtPCR results demonstrating deletion of full-length Wt1 in $W t 1^{\Delta / \Delta}$ MEFs. (C) Cell-cycle analysis of MEFs 7 days after infection with AdGFP (control) or AdCre. Numbers represent the percentage of cells in $\mathrm{G}_{1}$ (lower left), $\mathrm{S}$ (upper left) and $\mathrm{G}_{2} / \mathrm{M}$ (lower right) phase. (D) 3T3 assay

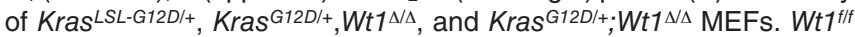
and Kras LSL-G12D/+;Wt1/f/f MEFs were also analyzed and performed similarly to $K$ ras $^{L S L-G 12 D /+}$ and $W t 1^{\Delta / \Delta}$ MEFs (data not shown). (E) Micrographs and quantitation of SA- $\beta$ gal staining of MEFs of indicated genotypes. Staining was performed 48 hours after plating of cells and 11 days after AdCre or AdGFP infection. Scale bars: $200 \mu \mathrm{m}$. (F) Immunofluorescence demonstrating increased levels of Pml-containing PNBs in

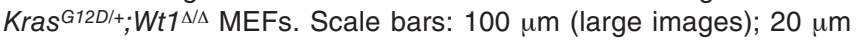
(insets). (G) Quantification of BrdU-positive cells 10 days after infection with AdCre. (H) Quantification of histone H3-positive cells 10 days after infection with AdCre. $P$ values are for a 2-tailed $t$ test. Error bars for all panels show mean \pm SD.

of cells entering mitosis (Figure 3, G and $\mathrm{H}$ ). Thus, $W t 1$ is a critical component of the genetic network that allows oncogenic Kras to induce proliferation and bypass senescence in primary cells.

Oncogenic Kras induces senescence in MEFs when it is expressed at high levels (34) and the mechanism of induction of senescence is due to a marked increase of Erk phosphorylation $(35,36)$. In contrast, expression of oncogenic Kras at physiologic levels neither induces senescence nor increases phosphorylation of Erk (25). Sprouty1 and MKP3, negative regulators of Mek/Erk signaling, have been reported as transcriptional targets of WT1 (37, 38). Therefore, Wt1 deletion might act by altering the intensity of Mek/Erk signaling and thereby altering the cellular response to oncogenic Kras. However, no consistent significant difference in the phosphorylation of Erk was found in $\mathrm{Kras}^{\mathrm{G} 12 \mathrm{D} /+}$; Wt $1^{\Delta / \Delta} \mathrm{MEFs}$ compared with Kras ${ }^{G 12 D /+}$ MEFs expressing normal levels of Wt 1 . Time course analysis was performed in 2 independent MEF lines (Supplemental Figure 4A and data not shown). Similar results were seen using a p-Mek-specific antibody (data not shown).

Activation of $\mathrm{p}^{16^{\text {Ink }} 4 \mathrm{a}}$ or $\mathrm{p} 19^{\text {Arf }}$ is frequently seen in association with senescence in mouse and human cells. Therefore, the level of expression of these proteins in $\mathrm{Kras}^{G 12 \mathrm{D} /+} ; \mathrm{Wt}^{\mathrm{\Delta}}{ }^{\Delta / \Delta} \mathrm{MEFs}$ was assessed. We did not detect a difference in the expression of $\mathrm{p} 16^{\text {Ink4a }}$ or $\mathrm{p} 19^{\mathrm{Arf}}$ when comparing $\mathrm{Kras}^{\mathrm{G} 12 \mathrm{D} /{ }^{+}}$to $\mathrm{Kras}^{\mathrm{G} 12 \mathrm{D} /+}$; Wt $1^{\Delta / \Delta}$ MEFs at day 7 after infection. In both genotypes, $\mathrm{p} 16^{\mathrm{Ink} 4 \mathrm{a}}$ and $\mathrm{p} 19^{\mathrm{Arf}}$ were increased to a similar level (Supplemental Figure 4B). As trp53 is also a critical regulator of senescence, we evaluated whether trp53 protein level or activity was altered in $\mathrm{Kras}^{\mathrm{G} 12 \mathrm{D} /+} ; \mathrm{Wt} 1^{\Delta / \Delta} \mathrm{MEFs}$. No consistent increase in trp53 protein levels in $\mathrm{Kras}{ }^{\mathrm{G} 12 \mathrm{D} /+} ; \mathrm{Wt} 1^{\Delta / \Delta}$ MEFs compared with Kras $^{\text {G12D/+ }}$ MEFS could be detected. As with p16 ${ }^{\text {Ink4a }}$ and p19Arf, trp53 levels were increased to a similar level (Supplemental Figure 4C). Thus, the senescence phenotype induced by Wt1 loss in MEFs expressing oncogenic Kras involves modulation of genetic pathways either downstream or parallel to Erk, p16 $6^{\text {Ink4a }}$, p19Arf, and trp53. These results demonstrate that Kras can lead to senescence even in the absence of Erk upregulation and that trp53 upregulation by itself is not a sufficient trigger for senescence in the presence of Wt 1 .

Loss of Wt 1 drives extensive alterations in gene expression modules driven by oncogenic Kras. Gene expression microarrays were used to obtain a genome-wide view of the changes induced by loss of wildtype Wt1 activity. RNA for independent lines of wild-type $(n=6)$,
$\operatorname{Kras}^{G 12 D /+}(n=5), W_{t} 1^{\Delta / \Delta}(n=5)$, and $\operatorname{Kras}^{G 12 D /+} ; W_{t} 1^{\Delta / \Delta}(n=6) \mathrm{MEFs}$ was generated 7 days after AdCre. This time point was chosen to provide enough time to see changes in gene expression in response to Wt1 loss while avoiding analyzing cells that were already phenotypically senescent (as these were not seen until days 10-11). A heat map including all probes with more than a 2 -fold change across any of the 4 genotypes demonstrated large gene expression changes in both $\mathrm{Kras}^{\mathrm{G} 12 \mathrm{D} / \mathrm{H}^{+}}$and $\mathrm{Kras}^{\mathrm{G12D} /+} ; \mathrm{Wt} 1^{\Delta / \Delta}$ when compared with wild-type and $W t 1^{\Delta / \Delta}$ MEFs (Figure $4 \mathrm{~A}$ and Supplemental Table 4). Surprisingly, we were unable to identify many genes that were differentially expressed between $\mathrm{Kras}^{\mathrm{G12D} /+}$ and $\mathrm{Kras}^{\mathrm{G12D} /+} ; \mathrm{Wt}^{\Delta / \Delta} \mathrm{MEFs}$, with commonly accepted statistical cutoffs (2-fold change and $10 \%$ false discovery rate [FDR] $q$ value, as determined by Significance Analysis of Microarrays [SAM]).

These results suggested that the phenotype observed upon Wt1 loss is the result of subtle changes across genes in 1 or more pathways rather than large fold changes of expression across a few genes. To further assess this possibility, the Molecular Signatures Database (MSigDB), a large collection of curated gene sets, was used to query the Kras ${ }^{G 12 D /+}$ vs. Kras ${ }^{G 12 D /+} ; W t 1^{\Delta / \Delta}$ data set using Gene Set Enrichment Analysis (GSEA) (39). Fifty-seven gene sets were enriched in $\mathrm{Kras}^{\mathrm{G12D} /{ }^{+}}$MEFs with an FDR of less than 25\% (Supplemental Table 5). This included a gene set of glutamine metabolism genes, "HSA00251_GLUTAMATE METABOLISM" (KEGG curated glutamate pathway), as well as experimentally derived gene sets downregulated by glutamate or leucine starvation ("PENG_GLUTAMINE_DOWN" and "PENG_LEUCINE_DOWN") (Figure 4B and Supplemental Figure 5A). Notably, 3 experimentally derived, independent gene sets for $M y c$ pathway genes were upregulated in $\mathrm{Kras}^{\mathrm{G} 12 \mathrm{D} /{ }^{+}}$MEFs compared with Kras ${ }^{\mathrm{G} 12 \mathrm{D} /+} ; \mathrm{Wt} 1^{\Delta / \Delta}$ MEFs (normalized enrichment score [NES] $-1.8,-1.79$, and -1.72 ; all with $P<0.05$, Figure 4C and Supplemental Figure 5B). Enrichment of Myc target genes was further confirmed using 4 additional gene sets not included in MSigDB (40) (Supplemental Table 6). These 4 gene sets were also found to be upregulated in $\mathrm{Kras}^{\mathrm{G} 12 \mathrm{D} /+}$ MEFs compared with $\mathrm{Kras}^{\mathrm{G} 12 \mathrm{D} /+} ; \mathrm{Wt} 1^{\Delta / \Delta}$ (NES -1.97, -1.77, -1.75, and -1.72; all with $P<0.001$ ) (Supplemental Figure 5C). Myc is known to cooperate with Kras to drive oncogenesis and RAS effector pathways and also stabilize MYC by phosphorylation (41). A role for Myc in regulating glutamine metabolism has also recently been demonstrated (42, 43). The GSEA results suggest that Wt1 may function to modulate the output of Myc, perhaps by specifically regulating those genes involved in glutamine metabolism.

A WT1-regulated gene signature is prognostic of survival in Kras-dependent patients. If WT1 target genes are important regulators of RAS-driven oncogenesis, it would be expected that patients with activated oncogenic KRAS signaling would benefit from lower expression of WT1-regulated genes. In other words, human lung cancer patients with activated RAS but with decreased expression of WT1-regulated genes (low WT1 gene signature) would have a better prognosis than those human lung cancer patients with increased expression of these genes (high WT1 gene signature). To test this hypothesis, a list of genes most consistently associated with Wt1 loss in MEFs was identified using the prediction analysis of microarrays (PAM) algorithm (44). PAM identified a gene signature consisting of 100 genes (62 with decreased expression in $\mathrm{Kras}^{\mathrm{G} 12 \mathrm{D} /+} ; \mathrm{Wt} 1^{\Delta / \Delta} \mathrm{MEFs}$ and 38 with increased expression in $\left.\mathrm{Kras}^{\mathrm{G} 12 \mathrm{D} /+} ; \mathrm{Wt} 1^{\Delta / \Delta} \mathrm{MEFs}\right)$ that accurately distinguished samples that express $\mathrm{Kras}^{\mathrm{G} 12 \mathrm{D} /+}$ in the context of either wild-type Wt1 or Wt1 deletion (Figure 4D and Supplemental Table 7). 
A
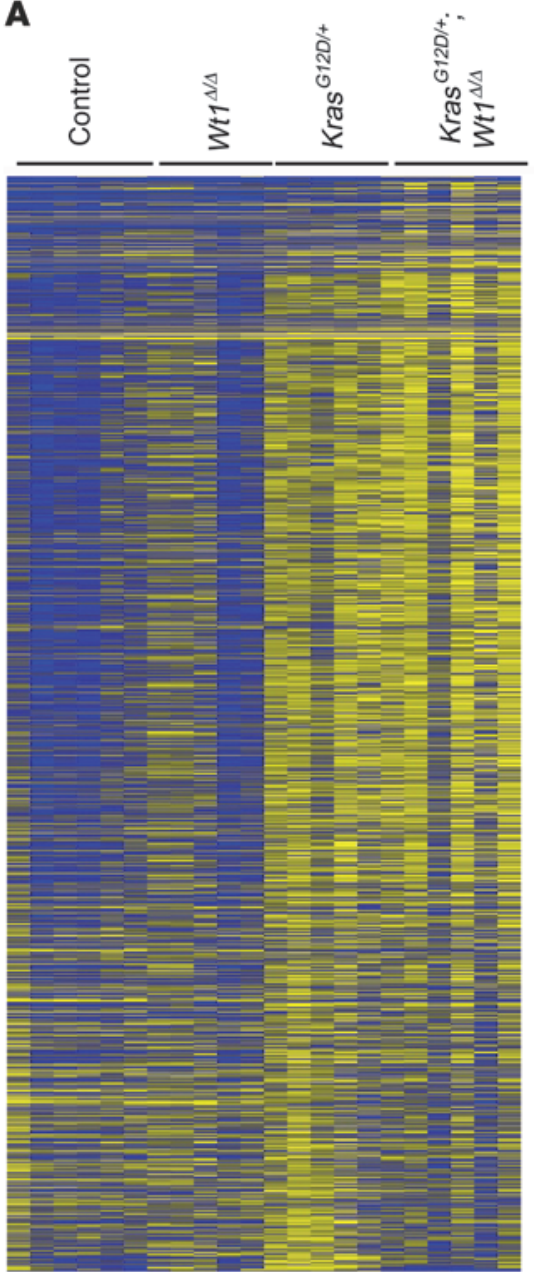

B Enrichment plot: PENG_GLUTAMINE_DN

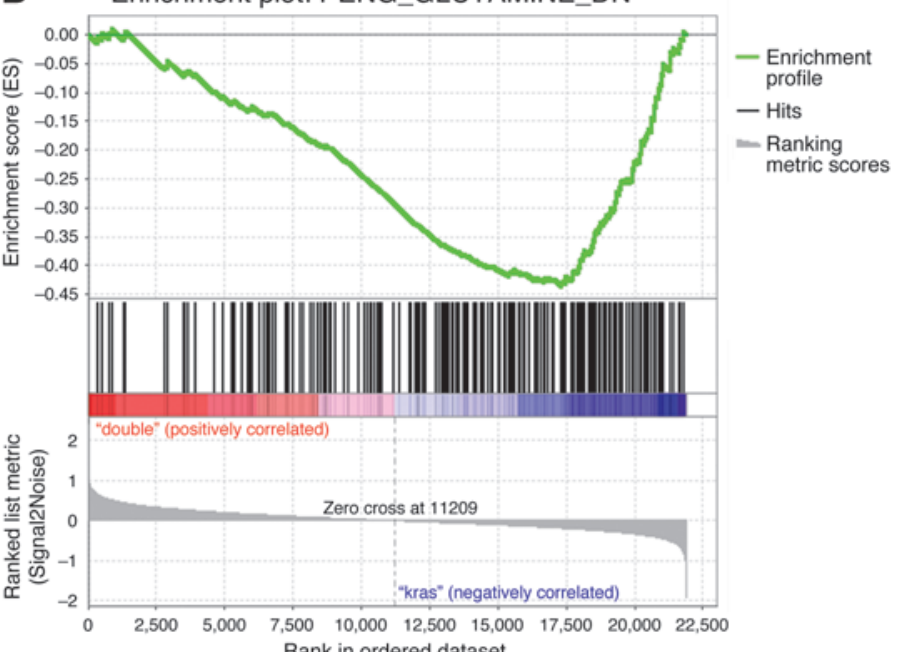

C Enrichment plot:SCHUMACHER_MYC_UP

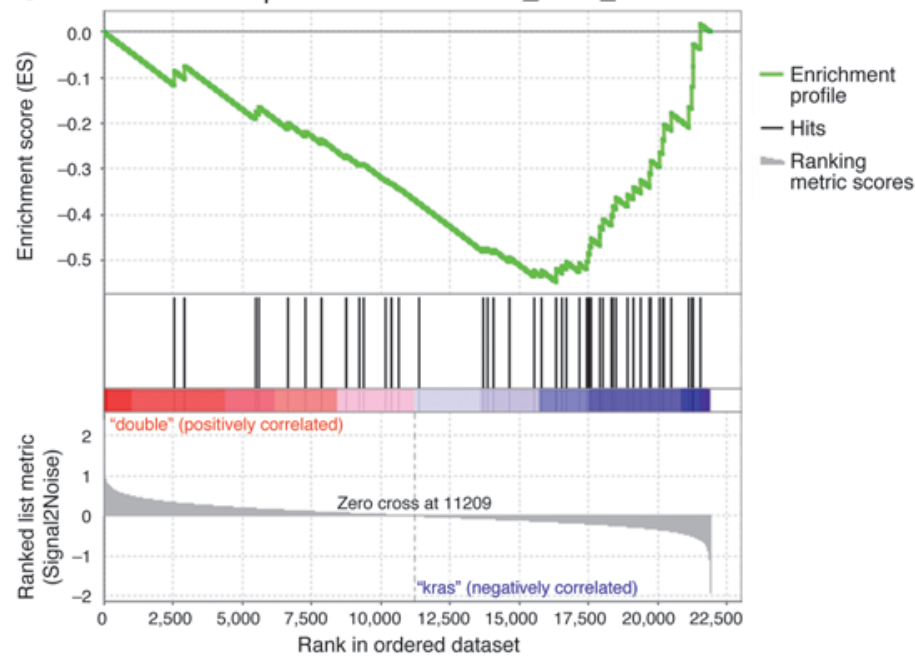

D

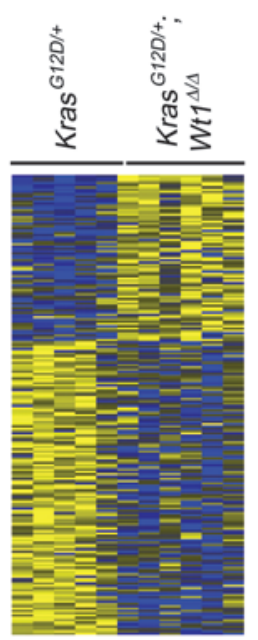

E

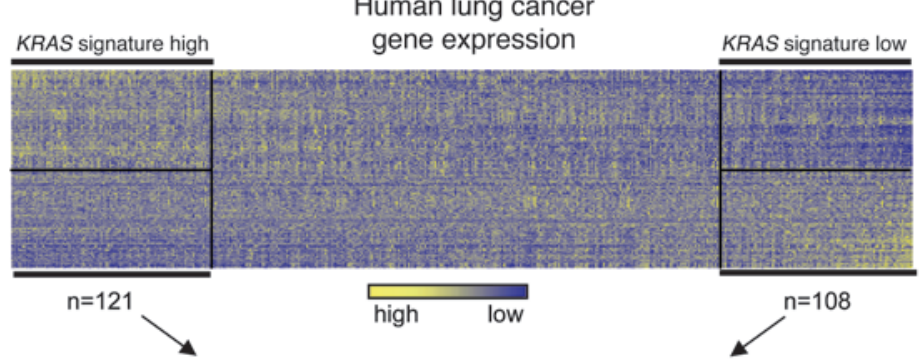

KRAS signature high
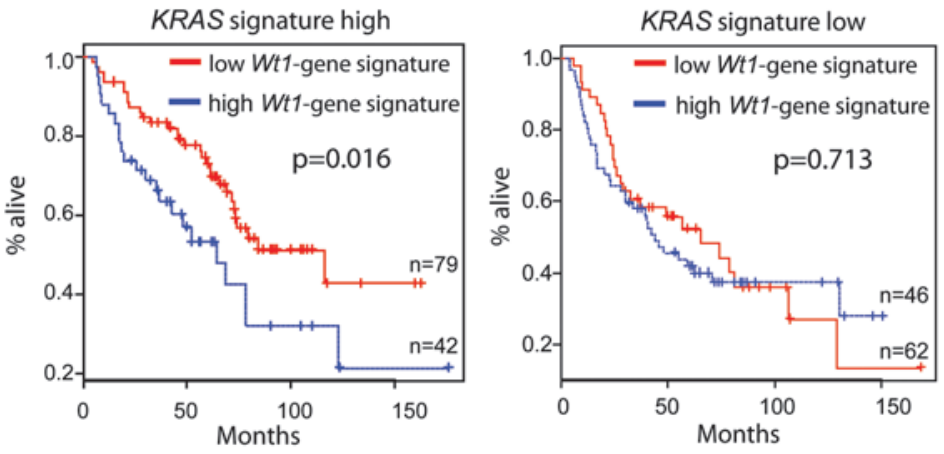


\section{Figure 4}

Wt1 loss leads to subtle alteration of gene expression in Kras-transformed MEFs. (A) Heat map of gene expression analysis of KrasG12D/+ and $K$ ras $^{G 12 D /+} ; W_{t 1}{ }^{\Delta / \Delta}$ MEFs compared with wild-type and Wt1 ${ }^{\Delta / \Delta}$ MEFs. Gene sets with at least a 2-fold change and an FDR of less than 0.1 for comparison between any of the 4 genotypes are shown. Enrichment plots of (B) MSigDB gene sets of glutamine metabolism genes as well as experimentally derived gene sets downregulated by glutamate or leucine starvation and (C) MSigDB gene sets for Myc pathway genes. (D) Heat map of differentially expressed genes between Kras ${ }^{G 12 D /+}$ and $K r a s^{G 12 D /+} ; W t 1^{\Delta / \Delta}$ MEFs as determined by PAM analysis. (E) A large gene expression compendium of human lung cancer gene expression (heat map) was analyzed in order to identify patients with either high expression or low expression of Kras signature genes. After training with $\mathrm{Kras}^{\mathrm{G} 12 \mathrm{D} /+} ; \mathrm{Wt1}^{\mathrm{\Delta} / \Delta}$ versus $\mathrm{Kras}^{\mathrm{G} 12 \mathrm{2D} /+}$ MEF data, high WT1 and low WT1 gene signature samples were identified from within the human lung carcinoma "KRAS signature high" and "KRAS signature low" groups. Kaplan-Meier analysis was used to assess survival of lung cancer patients with either high or low KRAS gene expression signatures as a function of the high/low WT1 gene signature. Log-rank $P$ values indicate differences between the 2 groups.

This gene set of WT1-regulated genes was then used to analyze a recently published large cohort of lung cancer gene expression data (45). As KRAS genotype is not available for this data set, an oncogenic KRAS gene expression signature was first derived using a smaller, independent data set of lung tumor gene expression for which the corresponding KRAS genotype is known (Supplemental Table 6) (46). This oncogenic KRAS signature was then used on the larger cohort in order to stratify patient samples as having high, low, or intermediate KRAS signature status (Figure 4E). After cross-species mapping (Supplemental Table 8), the MEF-derived "Wt1 high" and "Wt1 low" signature was used to stratify patients in the human lung cancer KRAS signature high and low groups into those with high WT1 or low WT1 gene signatures (see Supplemental Methods and Supplemental Tables 9 and 10). Importantly, the high WT1 or low WT1 gene signature was able to separate lung cancers that express KRAS signature genes into poor and good prognosis groups, respectively. In contrast, the high WT1 or low WT1 gene signature had no prognostic significance in samples without evidence of KRAS signature activation (Figure 4E). These results further support the hypothesis that genes whose expression is modulated by WT1 are specifically relevant to human lung cancers carrying oncogenic KRAS.

Decreased viability and increased senescence in mutant Kras human cancer cells after WT1 loss. To determine whether WT1 loss was deleterious to human cell lines expressing oncogenic KRAS, 2 shRNAs against human WT1 were used to knock down WT1 in a panel of human NSCLC lines with known RAS mutations status (Figure 5A). As expected, all KRAS mutant cells expressed high levels of KRAS protein (Supplemental Figure 6). Strikingly, a significant decrease in the cell viability of cell lines harboring KRAS mutations was observed in response to WT1 loss (Figure $5 \mathrm{~B})$. In contrast, a significantly lesser effect was seen in response to WT1 knockdown in NSCLC cell lines carrying wild-type KRAS. To extend this observation to other cell types in which KRAS mutations are present, we used a pair of human pancreatic cell lines with mutant (ASPC1) or wild-type (HPAFII) KRAS (Figure 5C). Consistent with the results in NSCLC, WT1 knockdown by 2 independent shRNAs lowered cell viability of ASPC1 cells to a significantly greater extent than HPAFII cells.
The genotype-specific effect of WT1 loss on cell viability was not due to increased apoptosis, as annexin V staining was not consistently different in cell lines expressing oncogenic Kras compared with those expressing wild-type Kras in response to WT1 knockdown (Figure 5D). In contrast, cell proliferation as measured by BrdU incorporation was significantly decreased in RAS mutant compared with RAS wild-type cell lines (Figure 5E). Mutant KRAS NSCLC cell lines adopted a senescence-like appearance in response to WT1 knockdown, and this was confirmed by staining with SA- $\beta$ gal (Figure 5, F and G). Thus, in response to WT1 loss, NSCLC cell lines expressing oncogenic KRAS showed a striking cell cycle arrest and senescence phenotype. Taken together, these results in human cells further support the role of WT1 as a regulator of proliferation in cells carrying RAS mutation.

Wt1 requirement for tumor progression in a mouse model of Kras-driven lung cancer. To assess the role of Wt 1 in Kras-driven tumor initiation and progression in vivo, Kras ${ }^{L S L-G 12 D /+}(n=10)$ and $\mathrm{Kras}^{L S L-G 12 D /+}$; $W_{t} 1^{f / f}(n=13)$ mice were generated and AdCre was delivered to the lung epithelium to induce simultaneous deletion of $\mathrm{Wt1}$ and activation of oncogenic Kras. Sixteen weeks after AdCre infection, deletion of Wt1 had a significant effect on tumor volume assessed both by microcomputed tomography (microCT) and histology (Figure 6, A and B). While some large tumors remained in Kras $^{L S L-G 12 D /+}$;Wt1f/f mice, PCR analysis of laser-capture microdissected tissue confirmed that in most cases those tumors retained at least 1 copy of Wt1 (Supplemental Figure 7). Analysis of tumor area excluding tumors with retention of a wild-type Wt1 allele (tumor area $>0.5 \mathrm{~mm}^{2}$ ) revealed a greater than $50 \%$ decrease of tumor area in $\mathrm{Kras}^{\mathrm{LSL}-G 12 \mathrm{D} /+}$; Wt1f/f compared with $\mathrm{Kras}^{\mathrm{LLL}-G 12 \mathrm{D} /+}$ mice (Figure 6C). Kras activation in the Kras ${ }^{L S L-G 12 D /+}$ tumor model led to the formation of multiple small adenomas, only a fraction of which would progress to adenocarcinoma. Analysis of total tumor number in $\mathrm{Kras}^{L S L-G 12 D /+}$ versus $\mathrm{Kras}^{L S L-G 12 D /+}$;Wt1f/f mice showed no significant differences (Figure 6D). However, binning of the total number of tumors in both genotypes into quartiles by size and assessing the percentage difference of the median size for each quartile revealed a gradual increase in the percentage difference from first to fourth quartile (Figure 6E). Median differences at each quartile were statistically significant between the 2 groups (Figure 6E). To assess whether this was due to changes in tumor cell proliferation, we stained lungs from $\operatorname{Kras}^{L S L-G 12 D /+}(n=4)$ and $\operatorname{Kras}^{L S L-G 12 D /+} ; W_{t} 1^{f / f}(n=4)$ mice with the proliferation marker Ki67. Tumors from Kras ${ }^{L S L-G 12 D /+}$; $W t 1^{f / f}$ mice had a significant decrease in the amount of Ki67-positive cells when compared with those from $\mathrm{Kras}^{L S L-G 12 D /+}$ mice (Figure $6 \mathrm{~F}$ ). These results strongly support a critical role for $W t 1$ in tumor progression of Kras-driven lung cancer in vivo.

\section{Discussion}

In this work, WT1 was identified as what we believe to be a novel modulator of oncogenic KRAS signaling. In both mouse and human cells, WT1 regulates the proliferative potential of oncogenic KRAS, and loss of WT1 drives cells expressing oncogenic KRAS toward a senescence program. In a primary screen and in 2 follow-up screens to identify genes that specifically function in mutant Kras cells, Wt1 shRNAs were negatively selected in the context of oncogenic Kras. These results were validated using a genetic approach in mice as well as utilizing shRNA-mediated knockdown in human cells. Surprisingly, these results demonstrate that physiologic levels of expression of oncogenic KRAS in the absence of WT1 lead to senescence. Activation of RAS signaling either by 
A

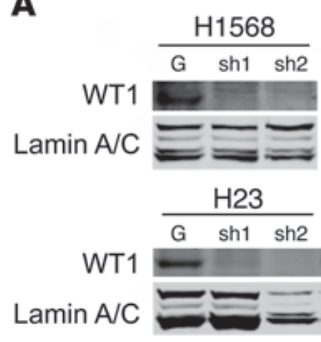

D

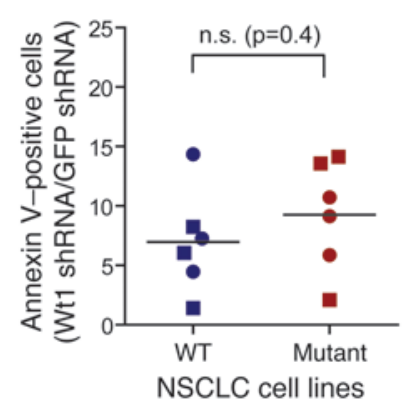

B

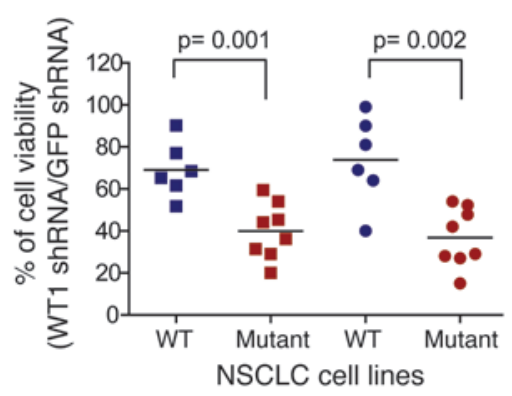

E

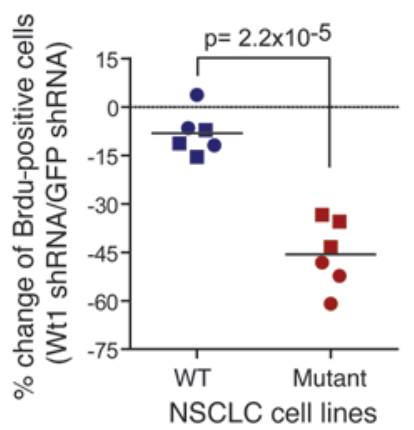

C WT1 ShRNA1 WT1 ShRNA2

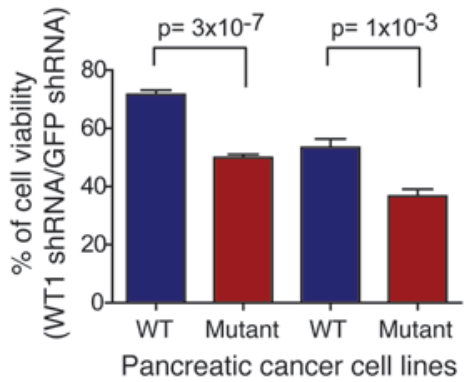

$\mathbf{F}$

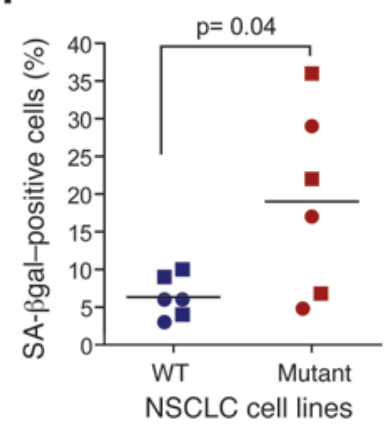

G

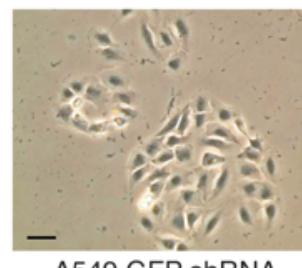

A549 GFP shRNA

$(<1 \%)$

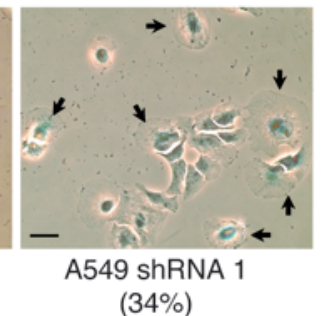

(34\%)

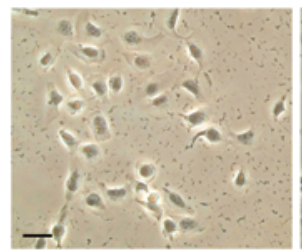

H1299 GFP shRNA $(<1 \%)$

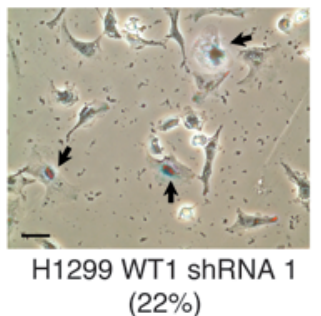

(22\%)

\section{Figure 5}

WT1 is a critical regulator of RAS-driven oncogenesis in human lung cancer. (A) Western blot showing WT1 suppression by 2 independent shRNAs in nuclear lysates of wild-type RAS NSCLC cells, NCl-H1568 (H1568), and mutant RAS NSCLC cells, NCl-H23 (H23). (B) Cell viability analysis of human NSCLC cell lines infected with WT1 shRNAs as measured by an MTT assay at day 5 after selection. RAS wild-type cell lines: NCl-H522, $\mathrm{NCl}-\mathrm{H} 1437, \mathrm{NCl}-\mathrm{H} 1568, \mathrm{NCl}-\mathrm{H} 1650, \mathrm{NCl}-\mathrm{H} 1975$, and NCl-H2126. RAS mutant cell lines: $\mathrm{NCl}-\mathrm{H} 23, \mathrm{NCl}-\mathrm{H} 358, \mathrm{NCl}-\mathrm{H} 441, \mathrm{NCl}-\mathrm{H} 460, \mathrm{NCl}-\mathrm{H} 727$, $\mathrm{NCl}-\mathrm{H} 1299, \mathrm{NCl}-\mathrm{H} 2009$, and NCl-A549. The same WT1 shRNAs were used for all experiments (squares: WT1 shRNA1; circles: WT1 shRNA2). Data points indicate percentage of cell viability (percentage of the ratio of WT1 shRNA-infected cells over GFP shRNA-infected cells). (C) Cell viability of pancreatic cancer cell lines with either wild-type (HPAF-II) or mutant KRAS (ASPC-1) after WT1 knockdown with the same shRNAs used in B. Results were normalized against cell viability of control cells transduced with a GFP shRNA. Error bars show mean \pm SD. (D) Annexin V analysis of NSCLC cell lines infected with 2 WT1 shRNAs or a GFP shRNA. Wild-type (NCI-H1568, NCl-H1975, and NCl-H2126) and mutant ( $\mathrm{NCl}-\mathrm{H} 23, \mathrm{NCl}-\mathrm{A} 549$, and NCl-H1299) RAS NSCLC cell lines were used. Data points show difference in annexin V staining in cells infected with WT1 shRNA compared with cells infected with control GFP shRNA. (E) Percentage change of BrdU-positive cells in NSCLC cell lines analyzed in D. Data points represent percentage change of BrdU incorporating cells between cells infected with WT1 shRNAs and control cells carrying a GFP shRNA. (F) Percentage of SA- $\beta$ gal-positive cells in NSCLC cells used in D. Data points indicate percentage of SA- $\beta$ gal-positive cells. (G) SA- $\beta$ gal staining of mutant RAS NSCLC cell lines described in D. Percentage of positive cells is shown in parentheses. Arrows point at senescence cells. Scale bars: $75 \mu \mathrm{m}$. All graphs are representative of at least 2 experiments. For all experiments, $P$ values are for a 2 -tailed $t$ test.

mutation of RAS proteins or loss of NF-1 can lead to senescence (5). Despite the potential importance of senescence as a barrier to tumorigenesis, the precise mechanisms accounting for the activation of senescence in response to RAS remain poorly understood. Furthermore, previous analysis of oncogenic KRAS signaling in primary cells suggested that perhaps senescence is an artifact of KRAS overexpression, leading to supraphysiologic activation of ERK, and that senescence does not occur under "physiologic" levels of oncogenic KRAS (25). In contrast, here we demonstrate that in mouse primary cells, senescence in response to oncogenic KRAS can occur despite a lack of upregulation of ERK signaling in specific contexts, i.e., in the absence of Wt 1 . The importance of Wt 1 expression was further demonstrated using human lung cancer cell lines and a mouse model of lung cancer. Wt1 deletion inhibits proliferation of Kras-driven lung tumors, thus inhibiting the progression of lung cancer. In addition, decreased expression of WT1-regulated genes is associated with improved prognosis in patients whose tumor gene expression profiles are consistent with activation of oncogenic 
A

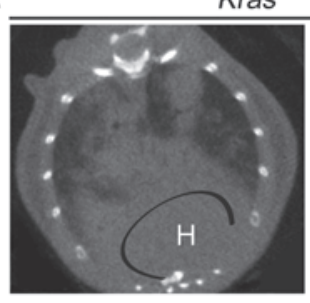

Kras $^{G 12 D /+}$

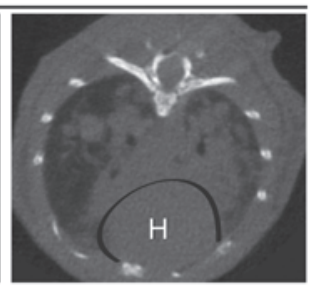

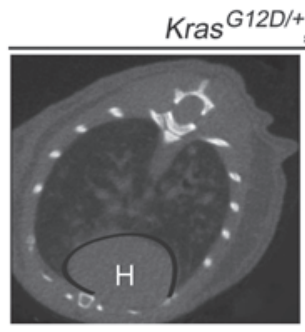

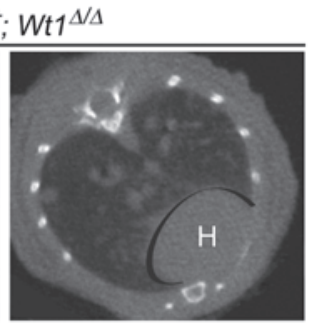

B

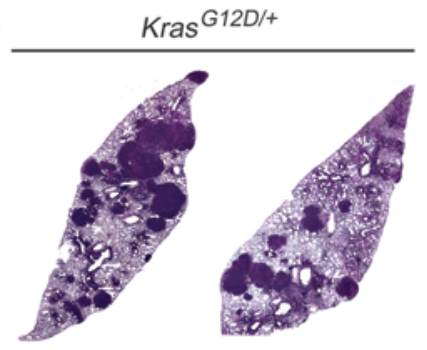

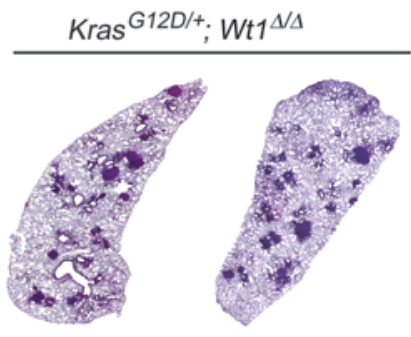

C

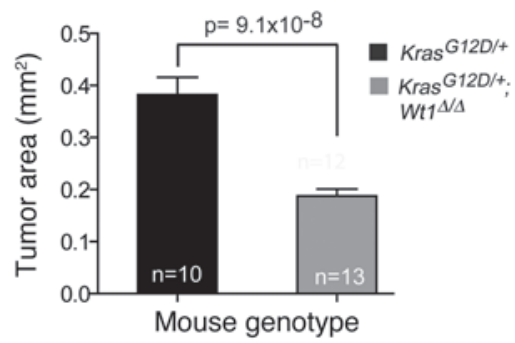

D

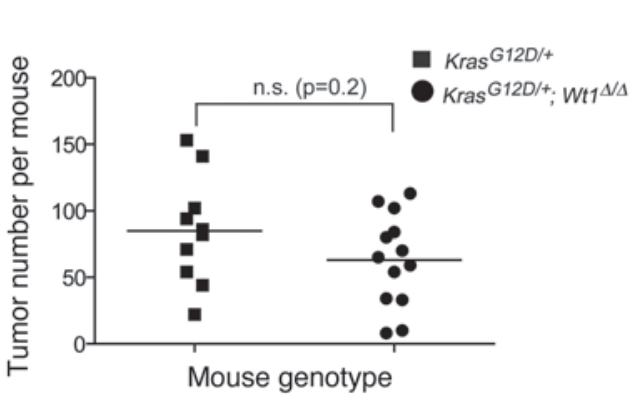

$\mathbf{E}$

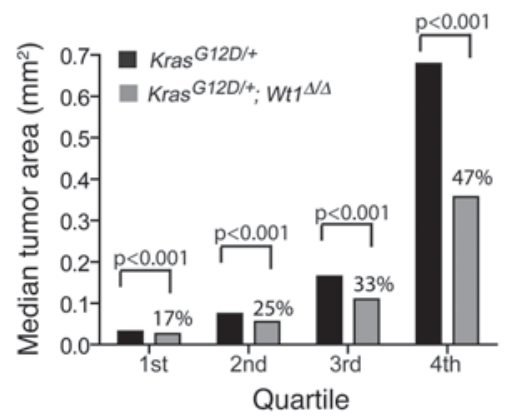

F
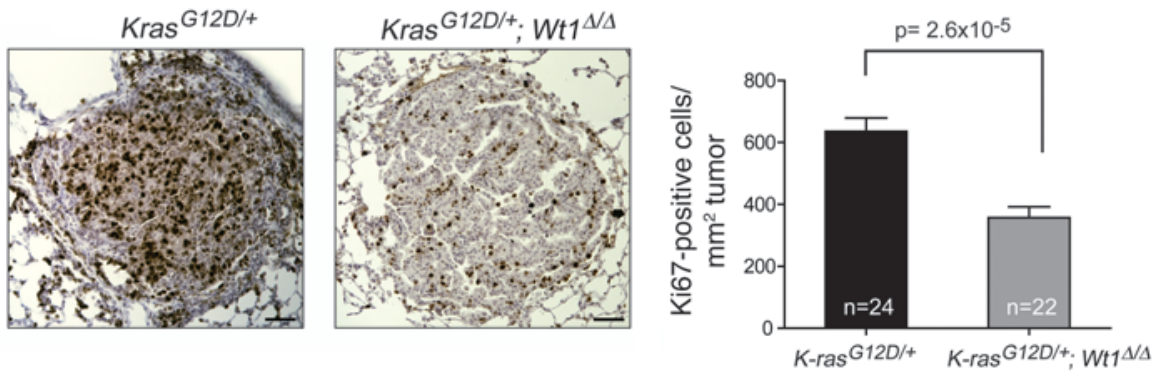

\section{Figure 6}

Wt1 deletion decreases lung tumor burden in vivo. (A) Representative microCT scans of tumors in Kras ${ }^{L S L-G 12 D /+}$ and $K r a s^{L S L-G 12 D /+} ; W_{t 1} 1^{t / f}$ treated with AdCre and allowed to develop tumors for 16 weeks. H. heart. (B) Representative H\&E-stained sections for Kras ${ }^{G 12 D /+}$ and $K r a s^{G 12 D /+}$;

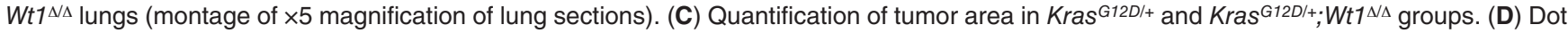
plot showing mean number of tumors per mouse in $\operatorname{Kras}^{L S L G 12 D /+}(n=10)$ and $K r a s^{G 12 D /+} ; W t 1^{\Delta / \Delta}(n=13)$ mice. Data points indicate total number of tumors per mouse. (E) Distribution of the total number of tumors into quartiles for Kras ${ }^{G 12 D /+}$ and $K r^{G} s^{G 12 D /+}$; Wt1 ${ }^{\Delta / \Delta}$ genotypes, based on their size. Median was calculated at each quartile, and $P$ values for the differences are indicated $(P<0.001)$. In addition, the percentage of the difference between the 2 groups was calculated. Percentage differences at each quartile are $17 \%$ (first), $25 \%$ (second), $33 \%$ (third), and $47 \%$ (fourth). (F) Representative images of tumors from Kras ${ }^{G 12 D /+}$ and $\mathrm{Kras}^{\mathrm{G} 12 \mathrm{D} /+} ; \mathrm{Wt1}^{\mathrm{\Delta} / \Delta}$ genotypes stained with the cell proliferation marker Ki67. Scale bars: $300 \mu \mathrm{m}$. Graph shows number of Ki67-positive cells per $\mathrm{mm}^{2}$ of tumor. Mice were infected with AdCre for 10 weeks, and all tumors included in

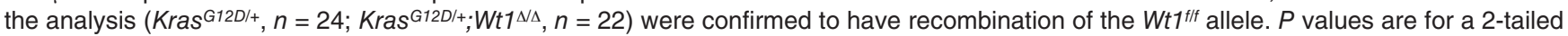
$t$ test. Error bars indicate mean \pm SEM.

KRAS. Taken together, these results using a combination of mouse and human cells demonstrate a surprising and unexpected role for WT1 as a critical regulator of the cellular mechanisms leading to either proliferation or senescence in response to oncogenic KRAS.
Several recent studies have utilized loss-of-function RNAi screens to identify genes that are selectively required in RAS-dependent human cell lines (18-20). Notably, WT1 loss was not identified as a synthetic-lethal interaction in the context of RAS in these studies. 
Negative selection screens are limited in their discriminative ability due to the noise inherent in any shRNA quantification method. Thus, in general, these screens are not saturating. As our screen was focused on fewer genes, this might explain why WT1 was identified whereas it was not in previously published work that included WT1 as part of the queried shRNA library (19). Another notable feature of the screen reported here is that it was performed in genetically defined murine cells. Previous work has demonstrated the utility of cross-species comparisons to leverage and filter gene expression profiles (11). This study provides further support for the utility of this cross-species approach by demonstrating that a signature derived from mouse primary cells with defined genetic mutations can be translated to lung cancer patients with oncogenic KRAS dependency as a predictor of overall survival. Thus, these results provide support for the use of functional screens using mouse shRNA libraries in order to study key molecular events in human oncogenesis.

While WT1 is best known as a tumor suppressor, considerable evidence points to a potential role for this protein as an oncogene in other tumor types (28). For example, almost all cases of the rare sarcoma desmoplastic small round blue cell tumor (DSRBCT) carry an EWS-WT1 translocation $(47,48)$. In addition, overexpression of WT1 has been reported in leukemias, breast, and lung cancer (49-51). WT1 has been demonstrated to play a role in regulation of apoptosis in a manner that is modulated by the serine protease HtrA2/Omi (52). Loss of HtrA2/Omi leads to increased WT1 protein levels, which confers resistance to apoptosis by cytotoxic drugs. Interestingly, in human lung cancer cell lines and in murine lung tumors, we detected very little apoptosis in response to WT1 depletion, suggesting that WT1 loss may have distinct consequences compared with WT1 upregulation. On the other hand, we observed that WT1 loss leads to senescence specifically in tumor cells harboring RAS mutations. Therefore, WT1 may function at least in part by ameliorating the oncogenic stress conferred by RAS mutations in these experimental systems. WT1 has also been shown to regulate expression of Snail at the transcriptional level, thus playing a key role in the differentiation toward mesenchymal lineages in embryonic stem cells and in the epicardium (53). Given these pleiotropic effects in normal and diseased cells, WT1 would appear to be a good candidate for a gene that can have "context-dependent" oncogenic or tumor suppressor effects (54). The complexity of the effect of WT1 overexpression and loss in normal and cancer cell physiology is perhaps partly due to the fact that WT1 has multiple isoforms with distinct biological functions. While some isoforms of WT1 are able to bind DNA and clearly have a role as transcription factors, other isoforms do not bind DNA but appear to participate in RNA processing. Further work will be needed to determine the specific targets of WT1 that are critical for regulating Ras-driven oncogenesis and senescence.

In conclusion, this work has identified what we believe to be a novel link between oncogenic KRAS signaling and expression of WT1. We used genomic data to inform a focused genetic screen with the goal of identifying regulators of oncogenesis. Our results demonstrate that the prooncogenic effects of WT1 are closely linked to elements of the KRAS signaling pathway. Further elucidation of this established link may provide avenues for therapeutic intervention in a wide range of human cancers. The general approach described here, combining computational analysis of expression signatures with cross-species functional genomics and genetics, should be applicable to other oncogenes and may provide insights into the complexity of the cancer genome.

\section{Methods}

shRNA library and virus production. The pLKO.1s lentiviral vector (gift of S. Stewart, Department of Cell Biology and Physiology, Washington University, St. Louis, Missouri, USA) was digested with EcoR1 to insert an oligonucleotide containing $\mathrm{Xma}$ and Nhe restriction sites. This vector was then digested with Xma and Nhe, and each of 100 oligonucleotides corresponding to the Luminex FlexMAP set (Luminex Corp.) was cloned into this site. The EcoRI site upstream of Xma was maintained and used to clone the shRNA hairpins together with the Age1 site upstream of the stuffer. Five shRNA hairpins against each of the genes of interest were designed using a publicly available algorithm (http://www.broad.mit.edu/science/ projects/rnai-consortium/trc-shrna-design-process). Then, 631 shRNAs against 162 candidate genes were cloned into the barcoded lentiviral vector (Supplemental Table 2) and sequence verified. Transfection-quality DNA was produced using a QIAGEN 96-well DNA kit. Vector DNA was quantitated and equimolar concentrations were pooled so that each "barcode" was represented only once in each pool. As the Luminex system detects 100 distinct barcodes, each pool had a maximum limit of 100 shRNAs (Supplemental Table 2). Pooled DNA was used to generate lentivirus and infect 2 mouse lung tumor cell lines (LKR10 and LKR13) at a low MOI so that on average each cell was infected with only 1 shRNA vector.

shRNA screen. LKR10 and LKR13 cells were infected in triplicate with 8 pools of approximately 100 shRNAs each for 2 days. Cells were selected with puromycin for 3 days and either passaged every 3-4 days or injected subcutaneously into immunodeficient mice and grown for 21 days. DNA was harvested at indicated time points for further analysis.

Lentiviral infections. Lentivirus was produced by transfection into $293 \mathrm{FT}$ cells as previously described (55), filtered, and applied directly to cells for infection at an MOI lower than 1.

Cell proliferation assay. Cells were plated into 96-well plates and treated with 3-[4,5-dimethlythiazol-2-yl]-2,5-diphenyl tetrazolium bromide (MTT). Experiments were read on the indicated days according to manufacturer's instructions (Cell Proliferation Kit I; Roche). All data were normalized to day 0 of experiment.

Apoptosis assay. Cell lines and MEFs were plated at $5 \times 10^{5}$ and $15 \times 10^{4}$ cells per $60 \mathrm{~cm}^{2}$ plates, respectively, and stained with annexin $V$ following the manufacturer's protocol (BD) at indicated time points. Annexin $\mathrm{V}$ analysis was done using FlowJo flow cytometry analysis software.

Cell-cycle analysis. Cell lines and MEFs were plated at $5 \times 10^{5}$ and $15 \times 10^{4}$ cells per $60 \mathrm{~cm}^{2}$ plates, respectively, and treated with $10 \mu \mathrm{M} \mathrm{BrdU}$ for 4 hours previous to analysis, fixed, and prepared for flow cytometry as previously described (56). Cell-cycle analysis was done using FlowJo flow cytometry analysis software.

Tumor formation assays. $25 \times 10^{3}$ LKR13 infected with specified shRNAs was suspended in $100 \mathrm{ml}$ of serum-free DMEM and injected subcutaneously into the 2 lower flanks of athymic Balb/c $\mathrm{c}^{\text {nu/nu }}$ mice (Charles River). One week after injection, tumor dimensions were measured every 3 days and tumor volume was calculated using the following: volume $=\pi / 6 \times($ length $) \times(\text { width })^{2}$.

Mouse embryo fibroblasts. Kras ${ }^{L S L-G 12 D}$ mice were crossed over 2 generations to $W t 1 f f f$ mice. MEFs were isolated from E13.5 embryos and genotyped by PCR. Early passage MEFs (p3-p4) were used for all experiments. All MEF experiments were done using $10 \%$ FCS/DMEM.

$3 T 3$ and senescence assays. 3T3 assays were as described (57). MEFs were infected with adenoviral GFP (AdGFP) or AdCre for 24 hours, allowed to grow for 1 day, and plated for $3 \mathrm{~T} 3$ assays. SA- $\beta$ gal staining assays were as described (58) and performed at day 11 after AdGFP or AdCre infection. For human NSCLC cell lines, SA- $\beta$ gal staining was done 11 days after infection with specific shRNAs as described in ref. 59. At least 5 random fields taken at $\times 20$ power using an inverted microscope were assessed to include a minimum number of 200 total cells per sample. 
Immunoblotting. Cells were scraped and lysed in $\mathrm{Mg} 2+\mathrm{Buffer}$ (for phospho-protein detection), NP-40 buffer (for p53 detection), or Laemmli buffer (for pml detection) supplemented with protease inhibitor cocktail (Roche), $25 \mathrm{mM}$ sodium fluoride, and $1 \mathrm{mM}$ sodium orthovanadate (Sigma-Aldrich). Protein samples were resolved by SDS-PAGE, transferred to Immobilon-FL membranes (Millipore), and incubated in blocking buffer (Li-Cor) for 1 hour prior to addition of primary antibody. Antibodies used were anti-mouse p53 (CM5; Novocastra), anti-PML (05-1708; Upstate), anti-p16 (M-156; Santa Cruz Biotechnology Inc.), anti-p21 (C-19; Santa Cruz Biotechnology Inc.), anti-K-ras (C-19; Santa Cruz Biotechnology Inc.), anti-Nras (C-20; Santa Cruz Biotechnology Inc.), anti-Hras (C-20; Santa Cruz Biotechnology Inc.), anti-Lamin A/C (636; Santa Cruz Biotechnology Inc.), anti-ERK (9102; Cell Signaling), and anti-p-ERK (9106; Cell Signaling). A WT1 antibody against the $\mathrm{C}$ terminus region of mouse Wt1 (GSDVRDLNALLPAVSSLGC) was produced and used for WT1 protein detection. Ras-GTP was pulled down with the Raf-1 Ras-binding domain (RBD) (17-218; Upstate). Following 3 TBS-T washes, infrared fluorescent-labeled secondary antibodies (IRDye 680 antirabbit or IRDye 800 anti-mouse; LI-COR) were incubated at room temperature for 1 hour and the membranes were scanned with the Odyssey Imaging Scanner (LI-COR). $\beta$-Actin antibody (clone 1A4; Sigma-Aldrich) was used as a loading control. p19 Arf immunoblotting was performed (Ab-80; Abcam) as previously described (60).

Immunofluorescence and immunohistochemistry. MEFs were trypsinized at day 9 after AdCre or AdGFP infection, plated onto slides, and allowed to grow overnight. Cells were fixed using 4\% PFA, processed, and stained with antibodies against pml (05-1708, Upstate) and BrdU (555627; BD). For quantitation, at least 5 random fields taken at $\times 40$ power were assessed to include a minimum number of 400 total cells per sample. Ki67 staining was performed as previously described (61).

rtPCR analysis. RNA was isolated 7 days after lentiviral infection using TRIzol reagent (Invitrogen) following the manufacturer's specifications. cDNA was synthesized with a DyNAmo cDNA synthesis kit (F470; New England Biolabs), and rt-PCR was performed using SYBR Green (Applied Biosystems) (see Supplemental Methods for primer sequences).

Gene expression analysis. RNA was isolated using TRIzOL 7 days after infection of MEFs with AdCre. RNA was further prepared by passage over an RNeasy column. cDNA synthesis, biotinylation of cRNA, and hybridization to mouse genome 4302.0 array was performed according to the manufacturer's instructions (Affymetrix). Microarray data was normalized with dChip software (http://biosun1.harvard.edu/complab/dchip/). Low signals (below 50) were filtered out using the PreprocessDataset module in GenePattern (http://www. broad.mit.edu/cancer/software/genepattern/). Differentially expressed genes in each group were identified using SAM (www-stat.stanford.edu/ tibs/SAM/) and Prediction Analysis for Microarrays (http://www-stat.stanford.edu/ tibs/ $\mathrm{PAM} /$ ). Genes with an FDR below $5 \%$ and fold change over 1.5 were included in the respective up/down lists. GSEA (http://www.broadinstitute.org/gsea/) (39) was used to compare our data sets with gene sets derived from previous studies. Raw data are available in Gene Expression Omnibus (GEO GSE15325).

microCT scans. Mice were anesthetized with $2 \%$ isoflurane and scanned using a GE Healthcare microCT scanner. Each scan was performed for 7 minutes with a resolution of $97 \mu \mathrm{m}$. Images were visualized using GE MicroView software.
Mice. Animals were intercrossed to generate $\mathrm{Kras}^{L S L-G 12 D /+}$ or $\mathrm{Kras}{ }^{L S L-G 12 D /+}$; $W_{t} 1^{f / f}$ animals. Mice were in a mixed $129 / \mathrm{Sv}$ and FVB background. Genotyping of mice was done on DNA extracted from tail clippings as described previously $(25,31)$. All animal experiments were approved by the Stanford University School of Medicine Committee on Animal Care (APLAC).

Tumor area analysis. Micrographs of H\&E slides from multiple lung sections for each sample were obtained and pictures were taken at $\times 20$ magnification. Bioquant software was used to montage the entire lung sections and to calculate tumor area for each sample. Tumor area was calculated by using the manual measurement feature in the Bioquant software. Tumor area was presented as $\mathrm{mm}^{2}$.

Cell lines. LKR10 and LKR13 were a gift of Julien Sage (Stanford School of Medicine, Stanford, California, USA) and were grown in DMEM supplemented with $10 \%$ FBS and $1 \%$ penicillin-streptomycin. MLE12 cells were purchased from ATCC and grown according to vendor's specifications. Human non-small cell lung cancer lines used either had wildtype RAS alleles (NCI-H522, NCI-H1437, NCI-H1568, NCI-H1650, NCIH1975, and NCI-H2126) or were mutant for RAS (NCI-H23, NCI-H358, NCI-H441, NCI-H460, NCI-H727, NCI-H1299, NCI-H2009, and NCIA549). All cell lines were grown according to ATCC specifications.

Statistics. A 2-tailed $t$ test was used for comparisons of different groups. Error bars correspond to either SD or SEM. $P<0.05$ was considered significant.

\section{Acknowledgments}

We thank Sheila Stewart for providing us with the $\mathrm{pLKO} .1 \mathrm{~s}$ vector. We thank Julien Sage, Steve Artandi, Laura Attardi, Kevin Haigis, Erica Jackson, and all the members of the Sweet-Cordero lab for helpful discussions. We thank the Stanford HighThroughput Biosciences Center and David Solow-Cordero for assistance in the execution of the pooled shRNA screen. We thank the members of the RNAi Platform at the Broad Institute and The RNAi Consortium (TRC) for advice and technical support. S. Vicent was supported by fellowships from the Fundación Alfonso Martín Escudero and the Tobacco-Related Disease Research Program of California (TRDRP). This work was supported in part by the NIH and the National Cancer Institute (I-K08CA98096-01 and 1R01CA138256-01 to E.A. Sweet-Cordero). E.A. Sweet-Cordero was also supported by grants from the Sidney Kimmel Foundation, the Rita Allen Foundation, the Joan's Legacy Foundation, and the Harold Amos Medical Faculty Development Program. W.C. Hahn was supported in part by the National Cancer Institute (R33 CA128625) and the Starr Cancer Consortium (I1-A11).

Received for publication June 28, 2010, and accepted in revised form August 25, 2010.

Address correspondence to: E. Alejandro Sweet-Cordero, 269 Campus Drive CCSR 1215B, Stanford, California 94305, USA. Phone: 650.725.5901; Fax: 650.736.0195; E-mail: ascor@stanford.edu.
1. Bos JL. ras oncogenes in human cancer: a review. Cancer Res. 1989;49(17):4682-4689.

2. Davies H, et al. Mutations of the BRAF gene in human cancer. Nature. 2002;417(6892):949-954.

3. Paez JG, et al. EGFR mutations in lung cancer: correlation with clinical response to gefitinib therapy. Science. 2004;304(5676):1497-1500.

4. Shaw AT, et al. Sprouty- 2 regulates oncogenic K-ras in lung development and tumorigenesis. Genes Dev. 2007;21(6):694-707.

5. Courtois-Cox S, et al. A negative feedback signaling network underlies oncogene-induced senescence. Cancer Cell. 2006;10(6):459-472.

6. Mooi WJ, Peeper DS. Oncogene-induced cell senescence--halting on the road to cancer. $N$ Engl J Med. 2006;355(10):1037-1046.

7. Collado M, Serrano M. Senescence in tumours: evidence from mice and humans. Nat Rev Cancer. 2010;10(1):51-57.

8. Arena S, Isella C, Martini M, de Marco A, Medico E, Bardelli A. Knock-in of oncogenic Kras does not transform mouse somatic cells but triggers a tran- scriptional response that classifies human cancers. Cancer Res. 2007;67(18):8468-8476.

9. Bild AH, et al. Oncogenic pathway signatures in human cancers as a guide to targeted therapies. Nature. 2006;439(7074):353-357.

10. Langenau DM, et al. Effects of RAS on the genesis of embryonal rhabdomyosarcoma. Genes Dev. 2007;21(11):1382-1395.

11. Sweet-Cordero A, et al. An oncogenic KRAS2 expression signature identified by cross-species geneexpression analysis. Nat Genet. 2005;37(1):48-55. 
12. Carro MS, et al. The transcriptional network for mesenchymal transformation of brain tumours. Nature. 2010;463(7279):318-325.

13. Lamb J, et al. A mechanism of cyclin $\mathrm{d} 1$ action encoded in the patterns of gene expression in human cancer. Cell. 2003;114(3):323-334.

14. Firestein R, et al. CDK8 is a colorectal cancer oncogene that regulates beta-catenin activity. Nature. 2008;455(7212):547-551.

15. Ngo VN, et al. A loss-of-function RNA interference screen for molecular targets in cancer. Nature. 2006;441(7089):106-110.

16. Schlabach MR, et al. Cancer proliferation gene discovery through functional genomics. Science. 2008;319(5863):620-624.

17. Silva JM, et al. Profiling essential genes in human mammary cells by multiplex RNAi screening. Science. 2008;319(5863):617-620.

18. Barbie DA, et al. Systematic RNA interference reveals that oncogenic KRAS-driven cancers require TBK1. Nature. 2009;462(7269):108-112.

19. Luo J, et al. A genome-wide RNAi screen identifies multiple synthetic lethal interactions with the Ras oncogene. Cell. 2009;137(5):835-848.

20. Scholl C, et al. Synthetic lethal interaction between oncogenic KRAS dependency and STK33 suppression in human cancer cells. Cell. 2009;137(5):821-834.

21. Campaner $\mathrm{S}$, et al. Cdk2 suppresses cellular senescence induced by the c-myc oncogene. Nat Cell Biol. 2010;12(1):54-59; sup 1-14

22. Ramaswamy $S$, et al. Multiclass cancer diagnosis using tumor gene expression signatures. Proc Natl Acad Sci U S A. 2001;98(26):15149-15154.

23. Osowski LD, Jakubek J, Woronkowicz M, Littleton N, KuKuruga D. The LUMINEX microbead array assay is an excellent sequence specific oligonucleotide probe (SSOP) method for HLA-A, B and DR antigen level typing. Hum Immunol. 2003;64:S97.

24. Johnson L, et al. Somatic activation of the K-ras oncogene causes early onset lung cancer in mice. Nature. 2001;410(6832):1111-1116.

25. Tuveson DA, et al. Endogenous oncogenic K-ras (G12D) stimulates proliferation and widespread neoplastic and developmental defects. Cancer Cell. 2004;5(4):375-387.

26. Kissil JL, et al. Requirement for Rac1 in a K-ras induced lung cancer in the mouse. Cancer Res. 2007;67(17):8089-8094.

27. Merkwirth C, et al. Prohibitins control cell proliferation and apoptosis by regulating OPA1-dependent cristae morphogenesis in mitochondria. Genes Dev. 2008;22(4):476-488.

28. Rivera MN, Haber DA. Wilms' tumour: connecting tumorigenesis and organ development in the kidney. Nat Rev Cancer. 2005;5(9):699-712.

29. Hammes A, et al. Two splice variants of the Wilms' tumor 1 gene have distinct functions during sex determination and nephron formation. Cell. 2001; 106(3):319-329.

30. Wikenheiser KA, et al. Production of immortalized distal respiratory epithelial cell lines from surfactant protein $\mathrm{C} /$ simian virus 40 large tumor antigen transgenic mice. Proc Natl Acad Sci U S A 1993;90(23):11029-11033.

31. Gao F, et al. The Wilms tumor gene, Wt 1 , is required for Sox 9 expression and maintenance of tubular architecture in the developing testis. Proc Natl Acad Sci U S A. 2006;103(32):11987-11992.

32. de Stanchina E, et al. PML is a direct p53 target that modulates p53 effector functions. Mol Cell. 2004;13(4):523-535.

33. Ferbeyre G, de Stanchina E, Querido E, Baptiste N, Prives C, Lowe SW. PML is induced by oncogenic ras and promotes premature senescence. Genes Dev. 2000;14(16):2015-2027

34. Serrano M, Lin AW, McCurrach ME, Beach D, Lowe SW. Oncogenic ras provokes premature cell senescence associated with accumulation of p53 and p16INK4a. Cell. 1997;88(5):593-602.

35. Zhu J, Woods D, McMahon M, Bishop JM. Senescence of human fibroblasts induced by oncogenic Raf. Genes Dev. 1998;12(19):2997-3007.

36. Lin AW, Barradas M, Stone JC, van Aelst L, Serrano $\mathrm{M}$, Lowe SW. Premature senescence involving p53 and p16 is activated in response to constitutive MEK/MAPK mitogenic signaling. Genes Dev. 1998;12(19):3008-3019.

37. Gross I, et al. The receptor tyrosine kinase regulator Sprouty 1 is a target of the tumor suppressor WT1 and important for kidney development.J Biol Chem. 2003;278(42):41420-41430.

38. Morrison DJ, Kim MK, Berkofsky-Fessler W, Licht JD. WT1 induction of mitogen-activated protein kinase phosphatase 3 represents a novel mechanism of growth suppression. Mol Cancer Res. 2008;6(7):1225-1231

39. Subramanian A, et al. From the Cover: Gene set enrichment analysis: A knowledge-based approach for interpreting genome-wide expression profiles. Proc Natl Acad Sci US A. 2005:102(43):15545-15550.

40. Zeller KI, Jegga AG, Aronow BJ, O’Donnell KA, Dang CV. An integrated database of genes responsive to the Myc oncogenic transcription factor: identification of direct genomic targets. Genome Biol. 2003;4(10):R69.

41. Yeh E, et al. A signalling pathway controlling c-Myc degradation that impacts oncogenic transformation of human cells. Nat Cell Biol. 2004;6(4):308-318.

42. Gao P, et al. c-Myc suppression of $\mathrm{miR}-23 \mathrm{a} / \mathrm{b}$ enhances mitochondrial glutaminase expression and glutamine metabolism. Nature. 2009; 458(7239):762-765

43. Wise DR, et al. Myc regulates a transcriptional program that stimulates mitochondrial glutaminolysis and leads to glutamine addiction. Proc Natl Acad Sci U S A. 2008;105(48):18782-18787.

44. Tibshirani R, Hastie T, Narasimhan B, Chu G. Diagnosis of multiple cancer types by shrunken centroids of gene expression. Proc Natl Acad Sci U S A. 2002;99(10):6567-6572.

45. Shedden K, et al. Gene expression-based survival prediction in lung adenocarcinoma: a multi- site, blinded validation study. Nat Med. 2008; 14(8):822-827.

46. Bhattacharjee A, et al. Classification of human lung carcinomas by mRNA expression profiling reveals distinct adenocarcinoma subclasses. Proc Natl Acad Sci U S A. 2001;98(24):13790-13795.

47. de Alava E, Ladanyi M, Rosai J, Gerald WL. Detection of chimeric transcripts in desmoplastic small round cell tumor and related developmental tumors by reverse transcriptase polymerase chain reaction. A specific diagnostic assay. Am J Pathol. 1995; 147(6):1584-1591.

48. Ladanyi M, Gerald W. Fusion of the EWS and WT1 genes in the desmoplastic small round cell tumor. Cancer Res. 1994;54(11):2837-2840.

49. Nakatsuka S, et al. Immunohistochemical detection of WT1 protein in a variety of cancer cells. Mod Pathol. 2006;19(6):804-814.

50. Yang L, Han Y, Suarez Saiz F, Minden MD. A tumor suppressor and oncogene: the WT1 story. Lenkemia. 2007;21(5):868-876.

51. Oji Y, et al. Overexpression of the Wilms' tumor gene WT1 in de novo lung cancers. Int J Cancer. 2002;100(3):297-303.

52. Hartkamp J, Carpenter B, Roberts SG. The Wilms' tumor suppressor protein WT1 is processed by the serine protease HtrA2/Omi. Mol Cell. 2010;37(2):159-171.

53. Martinez-Estrada OM, et al. Wt1 is required for cardiovascular progenitor cell formation through transcriptional control of Snail and E-cadherin. Nat Genet. 2010;42(1):89-93.

54. Rowland BD, Peeper DS. KLF4, p21 and contextdependent opposing forces in cancer. Nat Rev Cancer. 2006;6(1):11-23.

55. Rubinson DA, et al. A lentivirus-based system to functionally silence genes in primary mammalian cells, stem cells and transgenic mice by RNA interference. Nat Genet. 2003;33(3):401-406.

56. Brugarolas J, Chandrasekaran C, Gordon JI, Beach D, Jacks T, Hannon GJ. Radiation-induced cell cycle arrest compromised by p 21 deficiency. Nature. 1995;377(6549):552-557.

57. Sage J, et al. Targeted disruption of the three Rb-related genes leads to loss of G(1) control and immortalization. Genes Dev. 2000;14(23):3037-3050

58. Dimri GP, et al. A biomarker that identifies senescent human cells in culture and in aging skin in vivo. Proc Natl Acad Sci U S A. 1995;92(20):9363-9367.

59. Ansieau S, et al. Induction of EMT by twist proteins as a collateral effect of tumor-promoting inactivation of premature senescence. Cancer Cell. 2008;14(1):79-89.

60. Kamijo T, Weber JD, Zambetti G, Zindy F, Roussel MF, Sherr CJ. Functional and physical interactions of the ARF tumor suppressor with p53 and Mdm2. Proc Natl Acad Sci U S A. 1998;95(14):8292-8297.

61. Oliver TG, et al. Chronic cisplatin treatment promotes enhanced damage repair and tumor progression in a mouse model of lung cancer. Genes Dev. 2010;24(8):837-852. 